\title{
Diffusion-Enhanced Amide Bond Formation on a Solid Support
}

Johnny N. Naoum, Israel Alshanski, Agata Gitlin-Domagalska, Moshe Bentolila, Chaim Gilon and Mattan Hurevich 


\section{Table of Contents}

1. Materials …

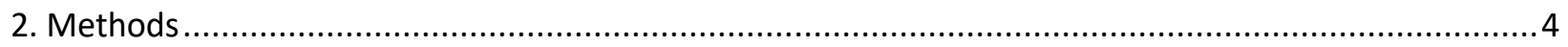



2.1.1 Schematic description of the HSS reactor setup........................................................ 4

2.1.2 Schematic description of the shaker reactor setup ...................................................... 4

2.2 General methodological approach for solid phase peptide synthesis (SPPS) .............................. 5

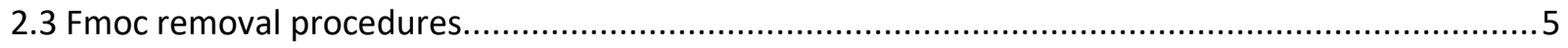

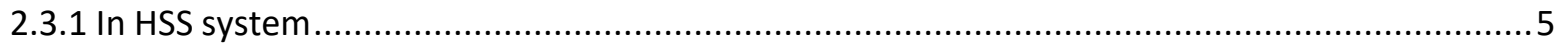

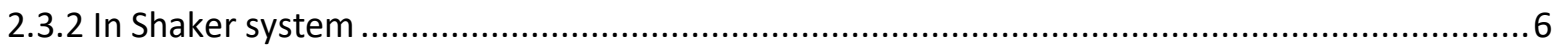

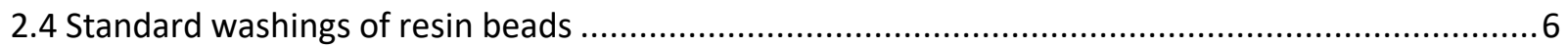

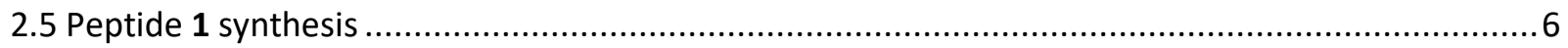

2.6 Coupling of Fmoc protected amino acid to peptide 1 by HSS at different mixing rates.................. 7

2.7 Coupling of Fmoc protected amino acid to peptide 1 without preactivation in a shaker ............... 7

2.8 Coupling of Fmoc protected amino acid to peptide 1 with preactivation by HSS......................... 8

2.9 Prolonged coupling of Fmoc protected amino acid to peptide 1 in a shaker ............................. 8

2.10 Prolonged coupling of Fmoc protected amino acid to peptide 1 by HSS.................................. 8

2.11 Prolonged preactivation time prior to coupling of Fmoc protected amino acid to peptide $\mathbf{1}$ by



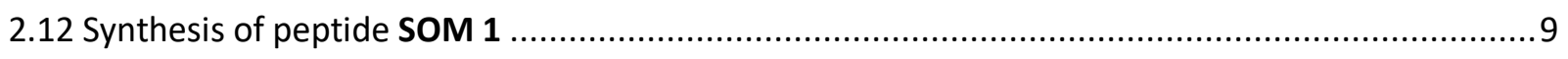

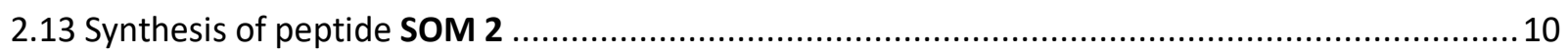

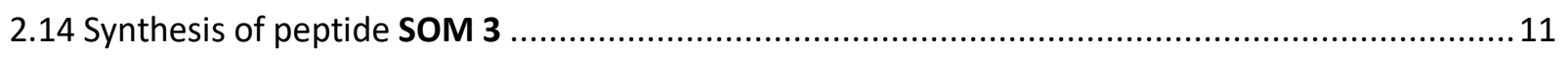

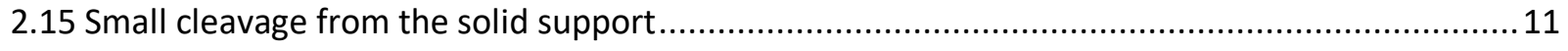

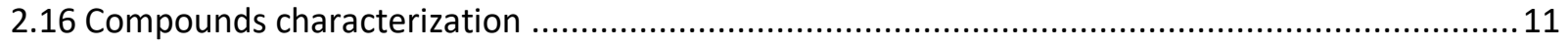

2.16.1 Analytical high performance liquid chromatography (HPLC)* ...................................... 11

2.16.2 Calculation of conversion from HPLC chromatogram .................................................. 12

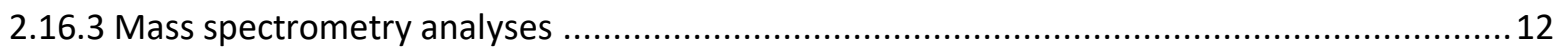

2.16.4 Microscope bead characterization details .................................................................. 13

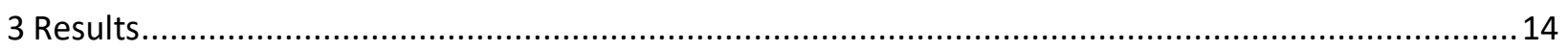

3.1 Time effect on the conversion of coupling of Fmoc His(Trt) 4a to peptide 1. ...........................14

3.2 Effect of prolonged preactivation time on coupling efficiency ............................................... 14

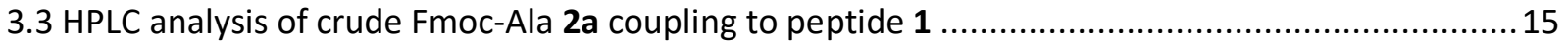

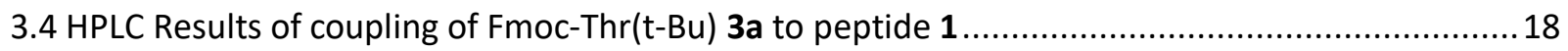


3.5 HPLC Results of coupling Fmoc-His(Trt) 4a to peptide 1

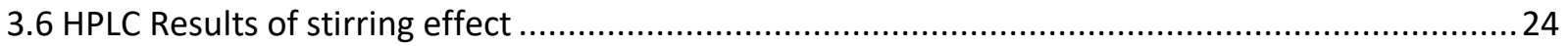

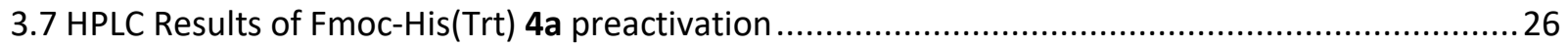

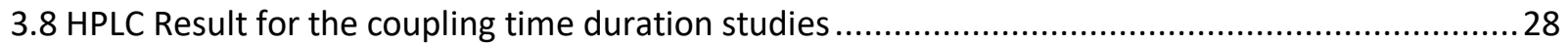

3.9 HPLC Results for the effect of duration during preactivation with HATU as coupling agent............30

3.10 HPLC Results for the effect of duration during preactivation with HBTU as coupling agent.........34

3.11 HPLC Results for the effect of duration during preactivation with COMU as coupling agent .......37

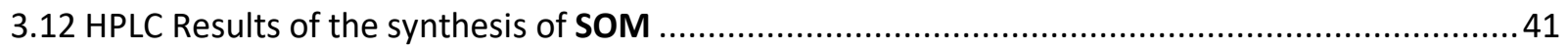

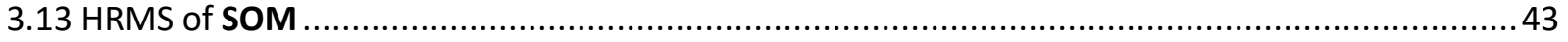

3.14 Microscope images of beads after applying HSS ................................................................ 44

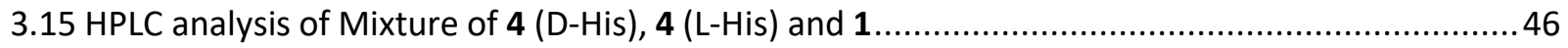




\section{Materials}

1-[Bis(dimethylamino)methylene]-1H-1,2,3-triazolo[4,5-b]pyridinium3-oxid

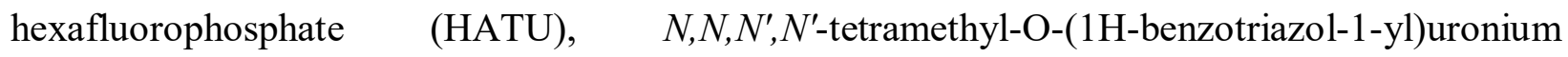
hexafluorophosphate (HBTU), (1-cyano-2-ethoxy-2-oxoethylidenaminooxy)dimethylaminomorpholino-carbenium hexafluorophosphate (COMU), and 9-fluorenylmethyloxycarbonyl- $N^{\alpha}$ protected amino acids (Fmoc- $\left.N^{\alpha}-\mathrm{XX}-\mathrm{OH}\right)$, were purchased from Chem-Impex International Inc. (Wood Dale, IL, U.S.A.). Fmoc-Rink-Amide methylbenzhydrylamine (MBHA) resin (200-400 mesh, $0.71 \mathrm{mmol} / \mathrm{g}$ resin) was purchased from Iris Biotech $\mathrm{GmbH}$ (Marktredwitz, Germany). Diethyl ether, piperidine, trifluoroacetic acid (TFA), $N, N$-diisopropylethylamine (DIPEA), methanol $(\mathrm{MeOH})$, triisopropylsilane (TIS), and other organic materials were purchased from ACROS ORGANICS N.V. (Geel, Belgium). Organic solvents for solid phase synthesis (SPS) and high performance liquid chromatography (HPLC) including: $N$-Methyl-2-pyrrolidone (NMP), dichloromethane (DCM), and acetonitrile (ACN) were purchased from J.T.Baker (NJ, U.S.A.).

\section{Methods}

\subsection{Mixing instrumentation}

2.1.1 Schematic description of the HSS reactor setup

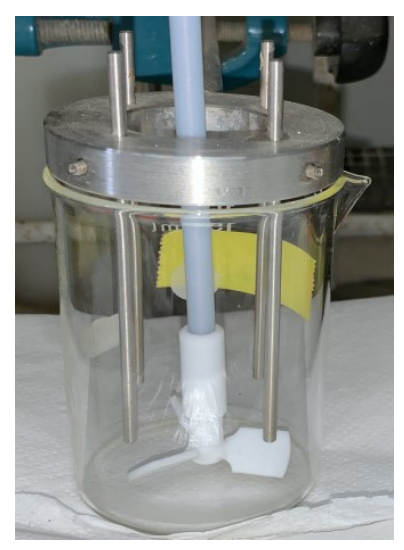

Figure S1. HSS setup containing: glass beaker, blade stirrer, and homemade adjustable baffles.

\subsubsection{Schematic description of the shaker reactor setup}




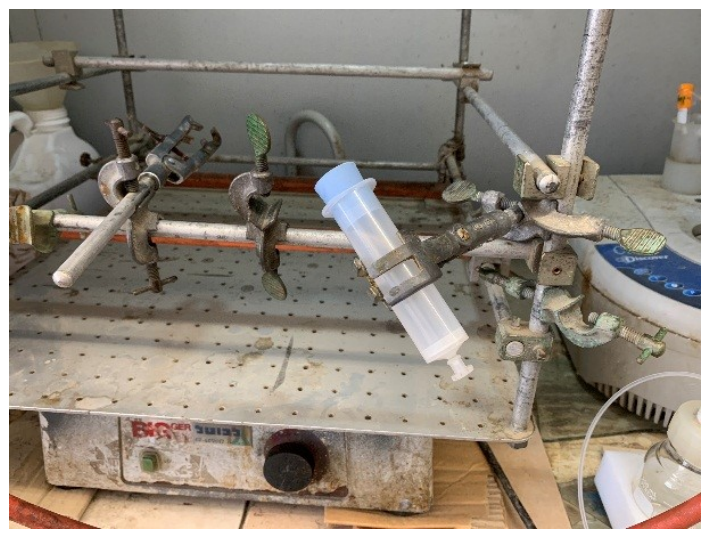

Figure S2. Shaker setup containing reaction vessel equipped with a sintered filter held by clamp on a rotatory orbital shaker.

\subsection{General methodological approach for solid phase peptide synthesis (SPPS)}

All peptides were synthesized on solid support from carboxy to amino terminus, and utilized Fmoc chemistry for the $N^{\alpha}$ protection. Fmoc Rink Amide methylbenzhydrylamine (Fmoc-MBHA) resin with loading capacity of $0.71 \mathrm{mmol} / \mathrm{g}$ was used for all the reactions in this work. The equivalents of all reagents used in solid phase synthesis (SPS) were calculated in respect to the resin loading capacity and weight. Resin beads were pre-swollen in NMP for 3-4 hours prior to any coupling protocol. Excluding the cleavage procedure, all other reactions on the solid support were performed under basic conditions utilizing DIPEA ( $\mathrm{pH} 8-9)$. The progress of each step in the synthesis was monitored by HPLC/MS following small cleavage procedure. All the experiments in this work were performed at room temperature.

\subsection{Fmoc removal procedures}

\subsubsection{In HSS system}

To the peptidyl-resin in the HSS reactor NMP was added and the suspension was poured into a fritted filter sep-pack cartridge (shown in Figure S2) connected to a vacuum pump until no beads are left in the reaction vessel. After washing the solid support (described in section 2.4), the bottom end of the cartridge was corked and a solution of $20 \%$ piperidine in NMP was added to the peptidyl-resin. The sep-pack cartridge was closed and shaken for 30 minutes. The solvents were filtered out and the reaction was repeated with a fresh solution of $20 \%$ piperidine in NMP for 30 minutes. At the end of the reaction the resin beads were washed with NMP (described in paragraph 2.4). 


\subsubsection{In Shaker system}

After the solid support was washed in a fritted filter sep-pack cartridge, a solution of $20 \%$ piperidine in NMP was added to the sep-pack and the reaction vessel was shaken for 30 minutes. The solvents were filtered out and the reaction was repeated with a fresh solution of $20 \%$ piperidine in NMP for 30 minutes. At the end of the reaction the resin beads were washed with NMP (described in paragraph 2.4).

\subsection{Standard washings of resin beads}

All washing steps were performed in a fritted filter sep-pack cartridge (shown in figure S2). After each reaction (either coupling or Fmoc removal) the reaction mixture was filtered out and the remaining resin beads were washed with SPPS organic solvents: NMP $(4 \times 2 \mathrm{~min})$ and DCM $(2 \times$ $2 \mathrm{~min})$.

\subsection{Peptide 1 synthesis}

The peptide was synthesized on a $3 \mathrm{~g}$ of peptidyl-resin in a sintered glass reaction vessel on a shaker system using standard Fmoc-SPPS chemistry and HATU/DIPEA as coupling system. Resin beads were swollen in NMP followed by Fmoc group removal (protocol 2.3.2). Fmoc-Ile-OH (3 equiv.) was dissolved in $15 \mathrm{~mL}$ NMP in a falcon tube and DIPEA (8 equiv.) was added. The solution was cooled to $0^{\circ} \mathrm{C}$ and a solution of HATU ( 3 equiv.) dissolved in $15 \mathrm{~mL}$ NMP was added. The final mixture was preactivated at $0^{\circ} \mathrm{C}$ and vortexed for 3 minutes prior to the addition to the reaction vessel. The reaction vessel was placed on the oval shaker and was mixed at $100 \mathrm{rpm}$ for 60 minutes. The peptidyl-resin was drained and the coupling reaction was repeated again. The resin was washed as described above and Fmoc was removed using the previously described protocol (section 2.3.2). The introductions of the following amino acids were performed by repeating the same coupling/Fmoc deprotection cycles as described for the Ile until the synthesis of the model peptide 1 ( $\mathrm{H}_{2} \mathrm{~N}$-Phe-Gly-Trp-Ile) was completed (Scheme $\left.\mathrm{S} 1\right)$. 

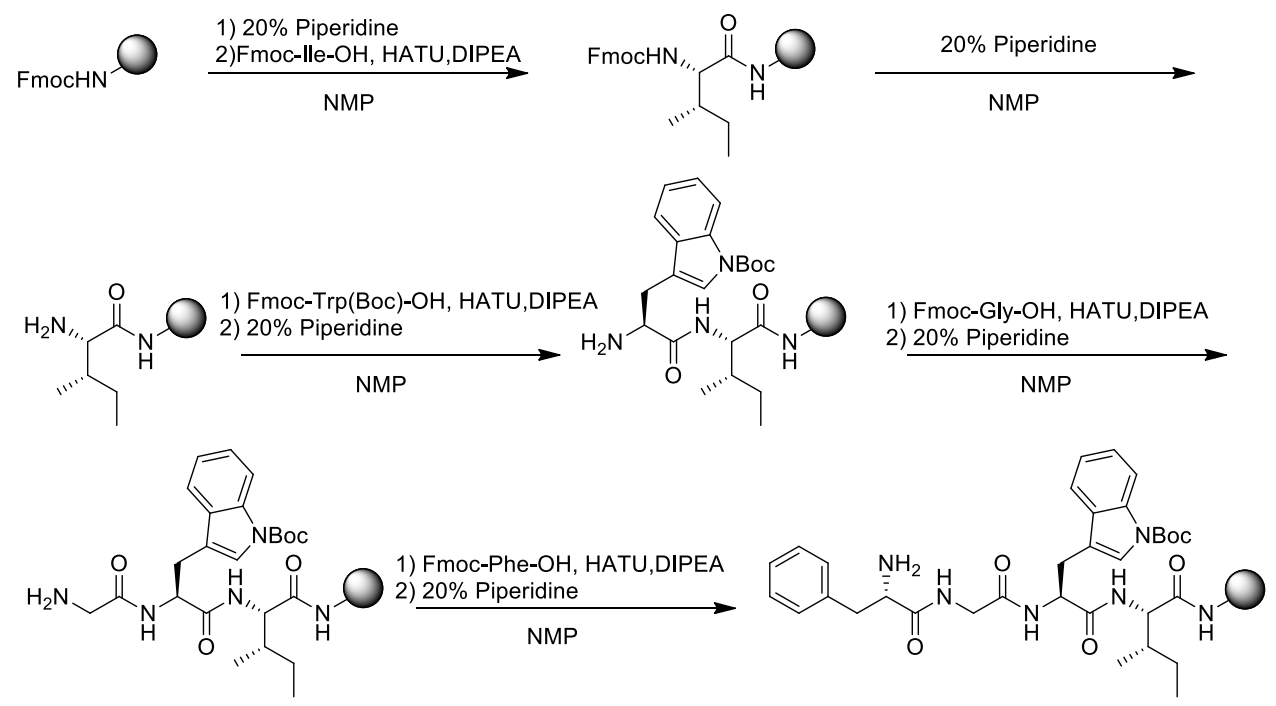

Scheme S1. Schematic synthesis of model peptide 1 on resin.

2.6 Coupling of Fmoc protected amino acid to peptide 1 by HSS at different mixing rates A $0.25 \mathrm{~g}$ of pre-swollen Peptide 1 resin was transferred to fritted filter sep-pack cartridge reaction vessel and the solvent was drained out. The sep-pack cartridge lower end was corked and $10 \mathrm{ml}$ NMP was added. This suspension was poured into the HSS reaction apparatus. The sep-pak cartridge was washed twice more with $10 \mathrm{ml}$ of NMP until all the resin was transferred to the HSS reaction beaker. To a solution of protected amino acid Fmoc- $N^{\alpha}$-His(Trt)-OH (1.1 equiv.) in 15 $\mathrm{mL}$ of NMP, DIPEA ( 8 equiv.) was added and the resulting mixture was poured into the HSS reaction apparatus. The suspension was mechanically stirred at 100,700 or $900 \mathrm{rpm}$ and a solution of HATU (1.1 equiv.) in $5 \mathrm{~mL}$ of NMP was added dropwise for over 1 minute without stopping the stirring. The reaction mixture was stirred at either 100, 700 or $900 \mathrm{rpm}$ for 60 minutes. At the end of the reaction the suspension was transferred to a fritted filter sep-pack cartridge, the solvent mixture was drained and the resin was washed as described in 2.4.

2.7 Coupling of Fmoc protected amino acid to peptide 1 without preactivation in a shaker A mixture of $0.05 \mathrm{~g}$ pre-swollen Peptide 1 resin in NMP was transferred to fritted filter sep-pack cartridge reaction vessel and the solvent was drained out. The sep-pack cartridge lower end was corked and a solution of protected amino acid Fmoc- $N^{\alpha}$-His(Trt)-OH (1.1 equiv.) in $5 \mathrm{~mL}$ NMP with DIPEA (8 equiv.) was poured into the cartridge. A solution of HATU (1.1 equiv.) in $5 \mathrm{~mL}$ NMP was added dropwise directly to the suspension in the reaction vessel for over 1 minute. The sep-pack cartridge top cap was stoppered and the reaction vessel was shaken using an oval shaker 
at $100 \mathrm{rpm}$ for 60 minutes. At the end of the reaction the solvent mixture was drained and the resin was washed as described in 2.4.

2.8 Coupling of Fmoc protected amino acid to peptide 1 with preactivation by HSS

A $0.25 \mathrm{~g}$ of pre-swollen Peptide 1 resin was transferred to fritted filter sep-pack cartridge reaction vessel and the solvent was drained out. The sep-pack cartridge lower end was corked and $10 \mathrm{~mL}$ of NMP was added. This suspension was poured into the HSS reaction apparatus. The sep-pack cartridge was washed twice more with $10 \mathrm{~mL}$ of NMP until all the resin was transferred to the HSS reaction beaker. To a solution of protected amino acid Fmoc- $N^{\alpha}$-His(Trt)-OH (1.1 equiv) in $15 \mathrm{~mL}$ NMP, DIPEA (8 equiv.) was added. To this mixture a solution of HATU (1.1 equiv.) dissolved in $5 \mathrm{~mL}$ NMP was added. The mixture was preactivated for 3 minutes and poured into the HSS reaction apparatus to reach a final volume of $50 \mathrm{~mL}$. The reaction mixture was stirred at $700 \mathrm{rpm}$ for 60 minutes. At the end of the reaction the suspension was transferred to a fritted filter sep-pack cartridge, the solvent mixture was drained and the resin was washed as described in 2.4.

\subsection{Prolonged coupling of Fmoc protected amino acid to peptide 1 in a shaker}

A solution of $0.05 \mathrm{~g}$ pre-swollen Peptide 1 resin in NMP was transferred to fritted filter sep-pack cartridge reaction vessel and the solvent was drained out. To a solution of protected amino acid Fmoc- $N^{\alpha}$-His(Trt)-OH (1.1 equiv.) in $5 \mathrm{~mL}$ NMP, DIPEA ( 8 equiv.) was added. To this mixture a solution of HATU (1.1 equiv.) dissolved in $5 \mathrm{~mL}$ NMP was added. The mixture was preactivated for 3 minutes and added to the reaction vessel. The reaction vessel was shaken for 16 hours. The reaction mixture was filtered out and the resin was washed as described in 2.4.

2.10 Prolonged coupling of Fmoc protected amino acid to peptide 1 by HSS

A $0.25 \mathrm{~g}$ of pre-swollen peptide 1 resin was transferred to fritted filter sep-pack cartridge reaction vessel and the solvent was drained out. The sep-pack cartridge lower end was corked and $10 \mathrm{~mL}$ of NMP was added. This suspension was poured into the HSS reaction apparatus. The sep-pak cartridge was washed twice more with $10 \mathrm{~mL}$ of NMP until all the resin was transferred to the HSS reaction beaker. To a solution of protected amino acid Fmoc- $N^{\alpha}$-His(Trt)-OH (1.1 equiv.) in 15 $\mathrm{mL}$ of NMP, DIPEA ( 8 equiv.) was added and this mixture was poured into the reaction apparatus to reach a volume of $45 \mathrm{~mL}$. The suspension was mechanically stirred at $700 \mathrm{rpm}$ and a solution of HATU (1.1 equiv.) in $5 \mathrm{~mL}$ NMP was added dropwise for over 1 minute without stopping the stirring. The reaction mixture was stirred at $700 \mathrm{rpm}$ for 120 minutes. At the end of the reaction 
the suspension was transferred to a fritted filter sep-pack cartridge, the solvent mixture was drained, and the resin was washed as described in 2.4.

2.11 Prolonged preactivation time prior to coupling of Fmoc protected amino acid to peptide 1 by shaker, with different coupling reagents

A solution of $0.05 \mathrm{~g}$ pre-swollen Peptide 1 resin in NMP was transferred to fritted filter sep-pack cartridge reaction vessel and the solvent was drained out. To a solution of protected amino acid Fmoc- $N^{\alpha}$-Ala-OH (1.5 equiv.) in $5 \mathrm{~mL}$ NMP, DIPEA (8 equiv.) was added. To this mixture a solution of HATU, HBTU or COMU (1.5 equiv.) dissolved in $5 \mathrm{~mL}$ NMP was added. The mixture was preactivated for $(1,5,10,20,40$, and 60) minutes and added to the reaction vessel. The reaction vessel was shaken for 60 minutes. The reaction mixture was filtered out and the resin was washed as described in 2.4.

\subsection{Synthesis of peptide SOM 1}

The peptide was synthesized utilizing Fmoc-SPPS chemistry. A solution of $0.05 \mathrm{~g}$ pre-swollen resin in NMP was transferred to fritted filter sep-pack cartridge reaction vessel and the solvent was drained followed by Fmoc group removal (protocol 2.3.2). The free amine function on resin was anchored with the first amino acid in the sequence, Fmoc-Gly-OH, as following: to a solution of protected amino acid Fmoc- $N^{\alpha}$-Gly-OH (1.5 equiv.) in $5 \mathrm{~mL}$ NMP, DIPEA (8 equiv.) was added. To this mixture a solution of HATU (1.5 equiv.) dissolved in $5 \mathrm{~mL}$ NMP was added. The mixture was preactivated for 3 minutes at $0^{\circ} \mathrm{C}$ and mixed using a vortex and added to the reaction vessel. The reaction vessel was shaken for 60 minutes at $100 \mathrm{rpm}$. The reaction solvent mixture was filtered out and the resin was washed as described in 2.4. The terminal amino acid was deprotected utilizing Fmoc group removal procedure (protocol 2.3.2). All coupling reactions were performed only once for each amino acid in the sequence. Fmoc removals and amino acid couplings were performed until the full planned somatostatin derived hexapeptide sequence Trp-Trp-Lys-ThrPhe-Gly-CONH 2 (Scheme S2) was formed. 

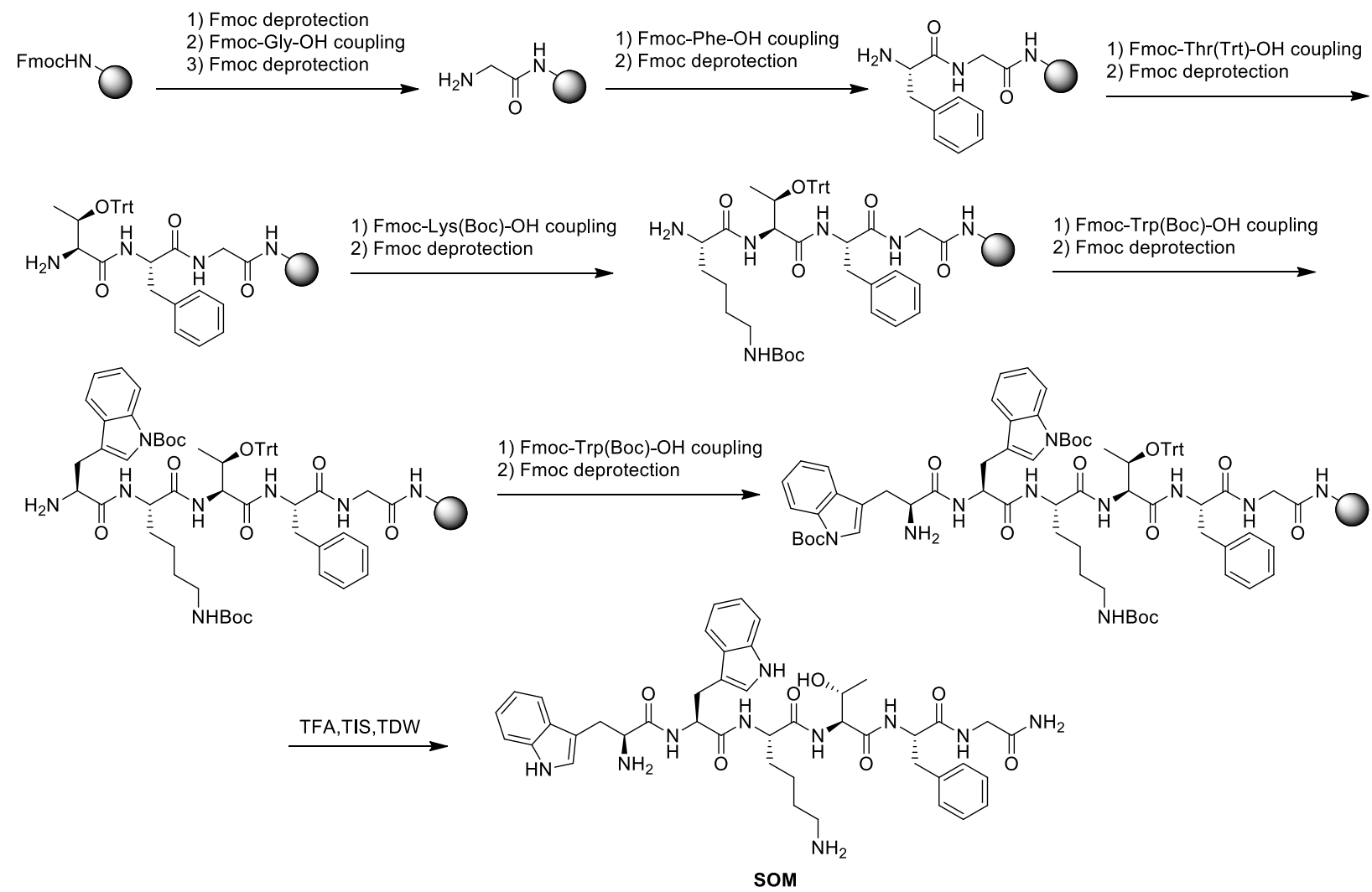

Scheme S2. Schematic synthesis of SOM on resin.

\subsection{Synthesis of peptide SOM 2}

The peptide was synthesized utilizing Fmoc-SPPS chemistry. A solution of 0.05 g pre-swollen resin in NMP was transferred to a fritted filter sep-pack cartridge reaction vessel and the solvent was drained followed by Fmoc group removal (protocol 2.3.2). The free amine function on resin was anchored with the first amino acid in the sequence, Fmoc-Gly-OH, as following: to a solution of protected amino acid Fmoc- $N^{\alpha}$-Gly-OH (3 equiv.) in 5 mL NMP, DIPEA (8 equiv.) was added. To this mixture a solution of HATU (3equiv.) dissolved in $5 \mathrm{~mL}$ NMP was added. The mixture was preactivated for 3 minutes at $0^{\circ} \mathrm{C}$ and mixed using a vortex and added to the reaction vessel. The reaction vessel was shaken for 60 minutes at $100 \mathrm{rpm}$. The reaction mixture was filtered out and the resin was washed as described in 2.4. The terminal amino acid was deprotected utilizing Fmoc group removal procedure (protocol 2.3.2). All coupling reactions were performed only once for each amino acid in the sequence. Fmoc removals and amino acid couplings were performed until the full planned somatostatin derived hexapeptide sequence Trp-Trp-Lys-Thr-Phe-Gly$\mathrm{CONH}_{2}$ (Scheme S2) was formed. 


\subsection{Synthesis of peptide SOM 3}

The peptide sequence was synthesized utilizing Fmoc-SPPS chemistry. A $0.25 \mathrm{~g}$ of the pre-swollen resin was transferred to a fritted filter sep-pack cartridge reaction vessel and the solvent was drained out followed by Fmoc group removal (protocol 2.3.1). The sep-pack cartridge lower end was corked and $10 \mathrm{ml}$ NMP were added. This suspension was poured into the HSS reaction apparatus. The sep-pack cartridge was washed twice more with $10 \mathrm{ml}$ of NMP until all the resin was transferred to the HSS reaction beaker. The free amine function on resin was anchored with the first amino acid in the sequence, Fmoc-Gly-OH, as following: to a solution of protected amino acid Fmoc- $N^{\alpha}$-Gly-OH (1.5 equiv.) in $15 \mathrm{~mL}$ of NMP, DIPEA ( 8 equiv.) was added and this mixture was poured into the reaction apparatus to reach a volume of $45 \mathrm{ml}$. The suspension was mechanically stirred at $700 \mathrm{rpm}$ and a solution of HATU (1.5 equiv.) in $5 \mathrm{ml}$ NMP was added dropwise for over 1 minute without stopping the stirring. The reaction mixture was stirred for 60 minutes. The reaction mixture was filtered out and the resin was washed as described in 2.4. The terminal amino acid was deprotected utilizing Fmoc group removal procedure (protocol 2.3.1). All coupling reactions were performed only once for each amino acid in the sequence. Fmoc removals and amino acid couplings were performed until the formation of the full planned somatostatin derived hexapeptide sequence Trp-Trp-Lys-Thr-Phe-Gly-CONH 2 (Scheme S2).

\subsection{Small cleavage from the solid support}

Few dried beads of the peptidyl-resin were dissolved in a $2 \mathrm{ml}$ pre-cooled solution (at $0^{\circ} \mathrm{C}$ ) composed of TFA (95\%), three distilled water (TDW) $(2.5 \%)$, and TIS (2.5\%). The mixture was shaken for 30 minutes at room temperature. The resin beads were then filtered out and TFA was fully evaporated by a stream of nitrogen. The remained crude peptide was dissolved in $2 \mathrm{~mL}$ ACN/TDW (1:1), filtered, and analyzed using analytical HPLC/MS.

\subsection{Compounds characterization}

\subsubsection{Analytical high performance liquid chromatography (HPLC)*}


HPLC analyses were performed on a Waters e2695 system equipped with a pump, $2489 \mathrm{UV} / \mathrm{Vis}$ variable wavelength detector recording, and a column. Chromatograms were recorded at $280 \mathrm{~nm}$ at room temperature with a flow rate of $1 \mathrm{~mL} / \mathrm{min}$. The mobile phase consisted of solution A: TDW (0.1\% v/v TFA) and solution B: ACN (0.1\% v/v TFA). The detailed HPLC gradient program is presented below. The collected fractions were analyzed by MS. To obtain analytical HPLC chromatograms of crude peptides, all samples were dissolved in TDW/ACN 1:1 mixture, filtered through a $0.45 \mu \mathrm{m}$ PTFE filters and injected to a reversed phase analytical HPLC column of Waters $\left(\mathrm{XSELECT}^{\mathrm{TM}} \mathrm{CSH}^{\mathrm{TM}} 130 \AA \mathrm{C} 18,4.6 \mathrm{~mm} \times 150 \mathrm{~mm}, 3.5 \mu \mathrm{m}\right)$.

*HPLC was performed for crude samples directly after small cleavage procedure.

Analytical HPLC program

\begin{tabular}{ccc}
\hline Time $(\min )$ & \% TDW & \% ACN \\
\hline 0 & 95 & 5 \\
1 & 95 & 5 \\
6 & 78 & 22 \\
16 & 68 & 32 \\
20 & 5 & 95 \\
22 & 5 & 95 \\
24 & 95 & 5 \\
26 & 95 & 5
\end{tabular}

\subsubsection{Calculation of conversion from HPLC chromatogram}

In this study the conversion percentages were calculated according to the following equation:

$$
\text { Equation 1: } \% \text { Conversion }=\frac{\text { Prod }}{\text { Prod }+ \text { React }} \times 100 \%
$$

Where Prod represents the area under the product peak, and React represents the area under the reactant peak.

\subsubsection{Mass spectrometry analyses}

Mass spectra were gained on LCQ Fleet Ion Trap mass spectrometer (Thermo Scientific) utilizing electrospray ionization. For high resolution mass spectrometry (HRMS) analyses, the spectra were recorded on Agilent 6550 iFunnel Q-TOF LC/MS system. 
2.16.4 Microscope bead characterization details

A small portion of the resin beads after the synthetic reaction, washings, and drying was put on glass slide under the microscope (ZEISS Scope.A1 with AxioCamm ICc 3). The resin beads measured with Objectives zoom of 5 and 20. The measured beads are spherical and intact with approximate diameter of $100 \mu \mathrm{m}$. 


\section{Results}

3.1 Time effect on the conversion of coupling of Fmoc His(Trt) 4 a to peptide 1. Table S1.

\begin{tabular}{cccc} 
Entry & Mixing Method & Duration (hours) & Conversion^ $^{\wedge}(\%)$ \\
\hline $1 \mathrm{~s}$ & Shaker & 1 & 46 \\
$2 \mathrm{~s}$ & Shaker & 16 & 49 \\
$3 \mathrm{~s}$ & HSS & 1 & 63 \\
$4 \mathrm{~s}$ & HSS & 2 & 61
\end{tabular}

${ }^{\wedge}$ Conversion was measured after Fmoc removal and cleavage from the solid support. The experiments were performed as follows: Entry 1s- coupling with a shaker (described in experimental section in the manuscript), Entry 2s- section 2.9, Entry 3s- coupling with HSS (described in experimental section in the manuscript), and Entry 4s- section 2.10 .

\subsection{Effect of prolonged preactivation time on coupling efficiency}

The coupling of 1.5 MER of Fmoc-Ala 2a to peptide $\mathbf{1}$ was chosen as a model since it resulted in high conversions. To generalize the effect, the study was performed for three different activating

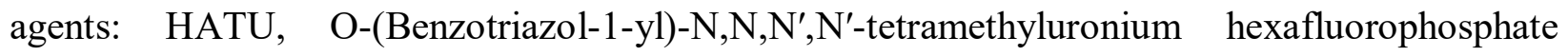
(HBTU), and (1-cyano-2-ethoxy-2-oxoethylidenaminooxy)dimethylamino-morpholinocarbenium hexafluorophosphate (COMU). The reactions were performed using a standard shaker changing only the duration of preactivation. There was a clear correlation between preactivation duration and the coupling efficiency.

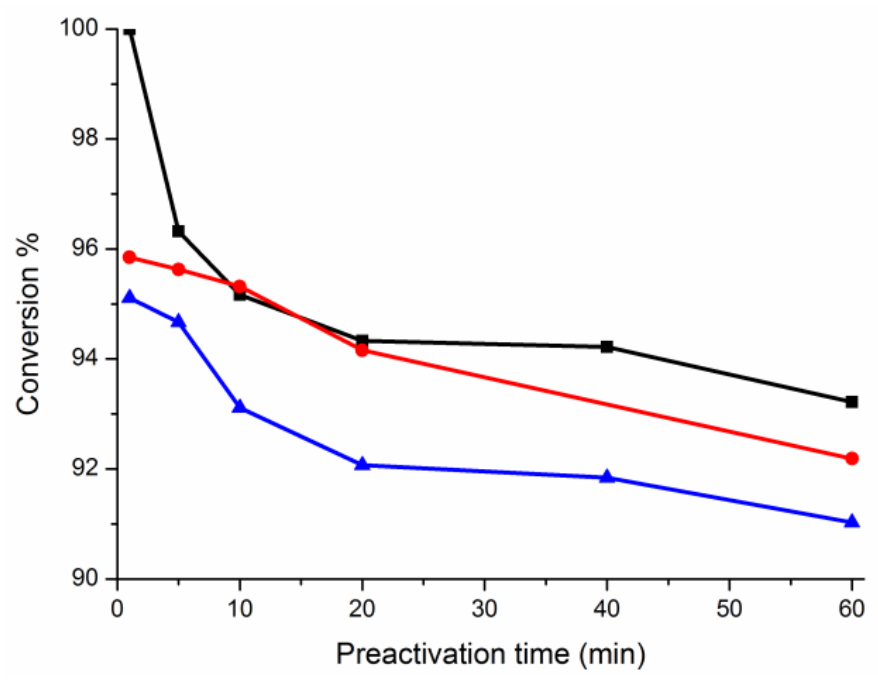

Figure S3. The effect of preactivation duration on the coupling efficiency. Coupling of Fmoc-Ala 2a to peptide 1 was performed after different duration of preactivation using the activation reagents HATU (Black), HBTU (Red) and COMU (Blue). The conversion was determined by HPLC. 


\subsection{HPLC analysis of crude Fmoc-Ala 2 a coupling to peptide 1}

Peak at 12.5 minutes corresponds to peptide $\mathbf{1}$ and peak at 13.3 minutes corresponds to the product 2. All chromatograms were recorded for crude samples after cleavage without further purification. Peaks after 18 minutes (designated by the vertical dashed line) are related to residual protecting group moieties and to the HPLC gradient program, which corresponds to washing region and do not contain the peptides that are relevant for this study.

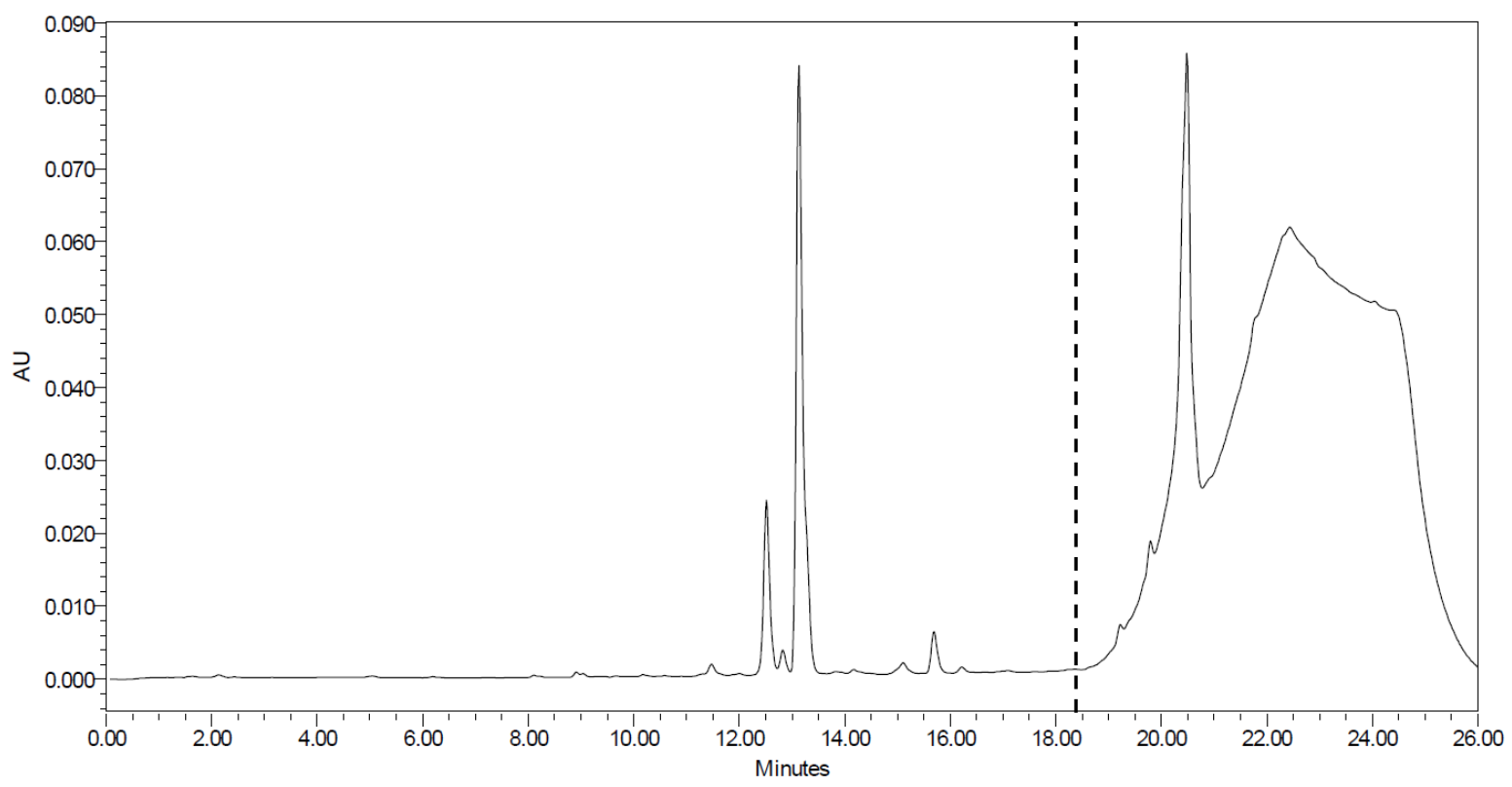

Figure S4. RP-HPLC Chromatogram of crude peptide obtained after coupling of Fmoc-Ala 2a to peptide 1 with 1.1 MER for one hour in a shaker at $100 \mathrm{rpm}$ (Table 1, Entry 1). 


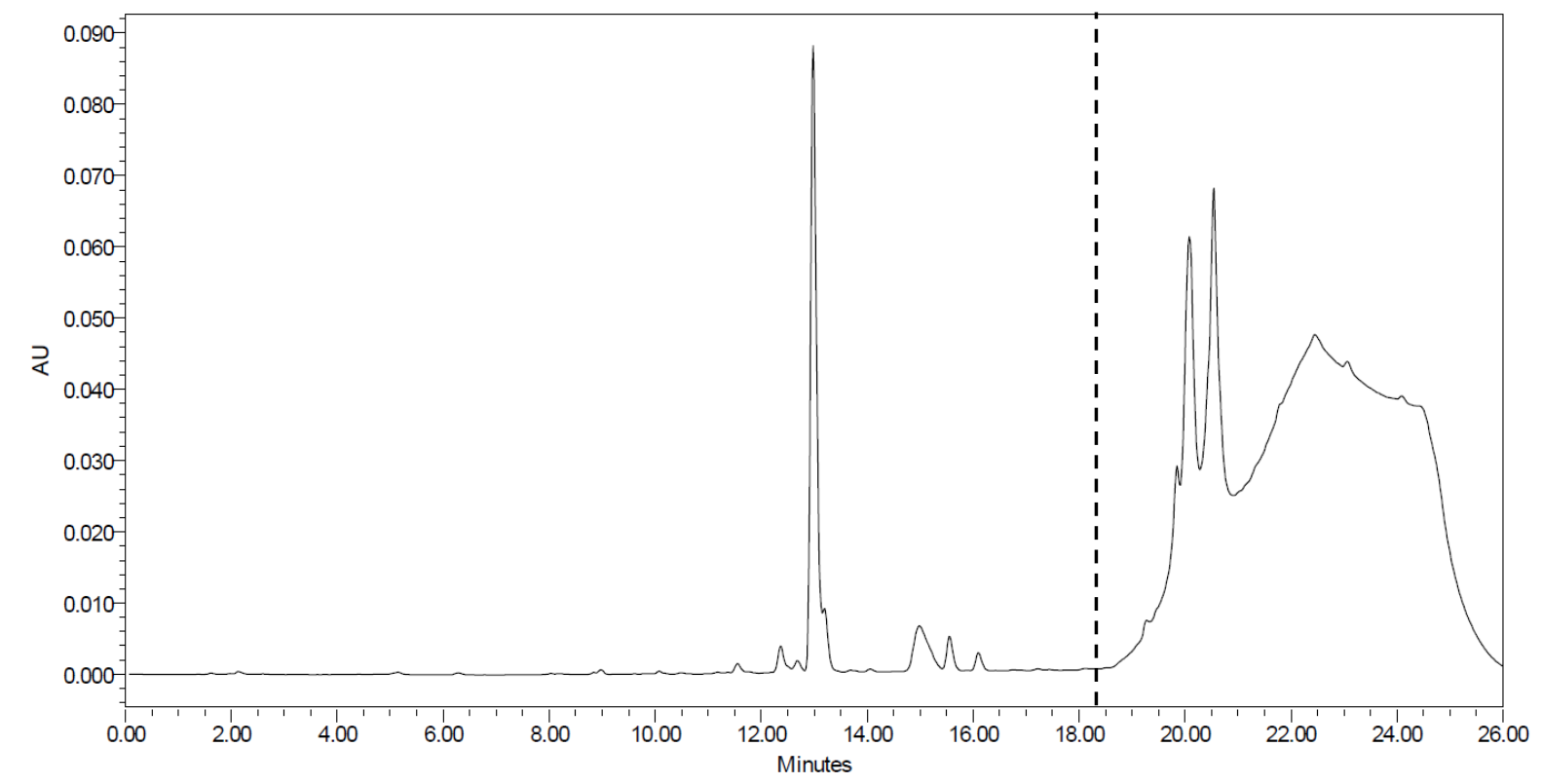

Figure S5. RP-HPLC Chromatogram of crude peptide obtained after coupling of Fmoc-Ala 2a to peptide 1 with 1.5 MER for one hour in a shaker at $100 \mathrm{rpm}$ (Table 1, Entry 3).

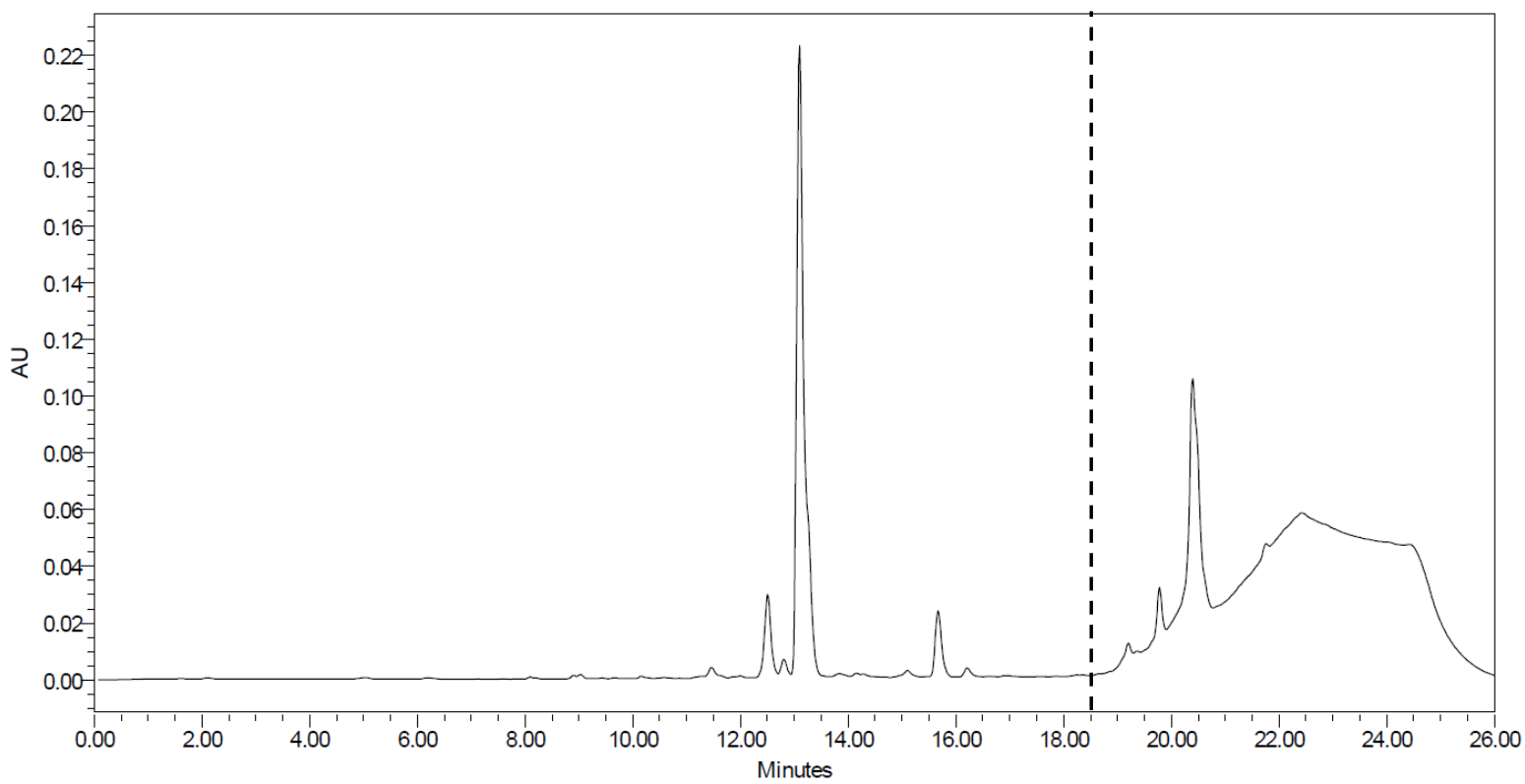

Figure S6. RP-HPLC Chromatogram of crude peptide obtained after coupling of Fmoc-Ala 2a to peptide 1 with 1.1 MER for one hour in HSS at $700 \mathrm{rpm}$ (Table 1, Entry 2). 


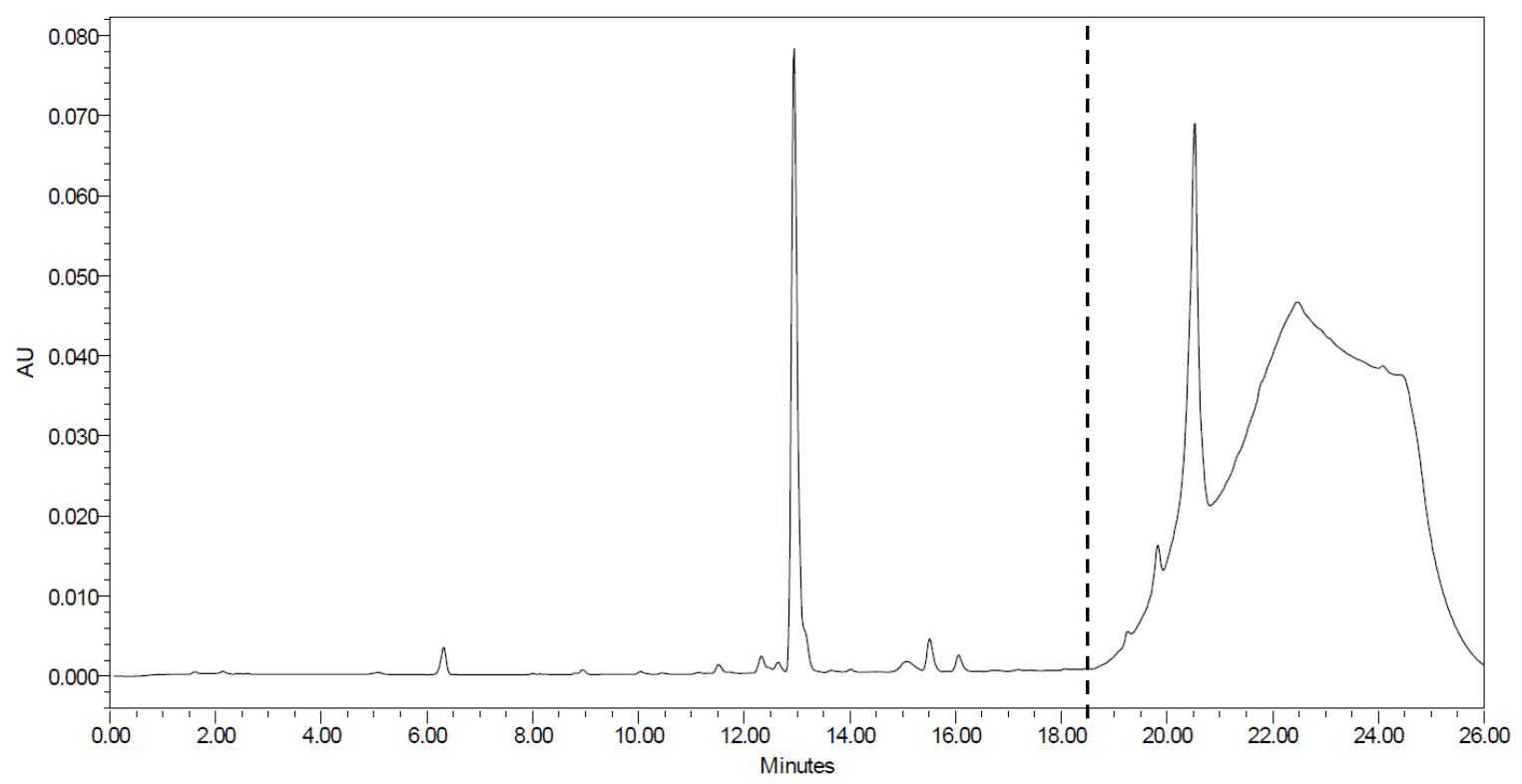

Figure S7. RP-HPLC Chromatogram of crude peptide obtained after coupling of Fmoc-Ala 2a to peptide 1 with 1.5 MER for one hour in HSS at $700 \mathrm{rpm}$ (Table 1, Entry 4). 


\subsection{HPLC Results of coupling of Fmoc-Thr(t-Bu) $3 a$ to peptide 1}

Peak at 12.4 minutes corresponds to peptide 1 and peak at 13 minutes corresponds to the product 3. All chromatograms were recorded for crude samples after cleavage without further purification. Peaks after 18 minutes (designated by the vertical dashed line) are related to residual protecting group moieties and to the HPLC gradient program which corresponds to washing region and do not contain the peptides that are relevant for this study.

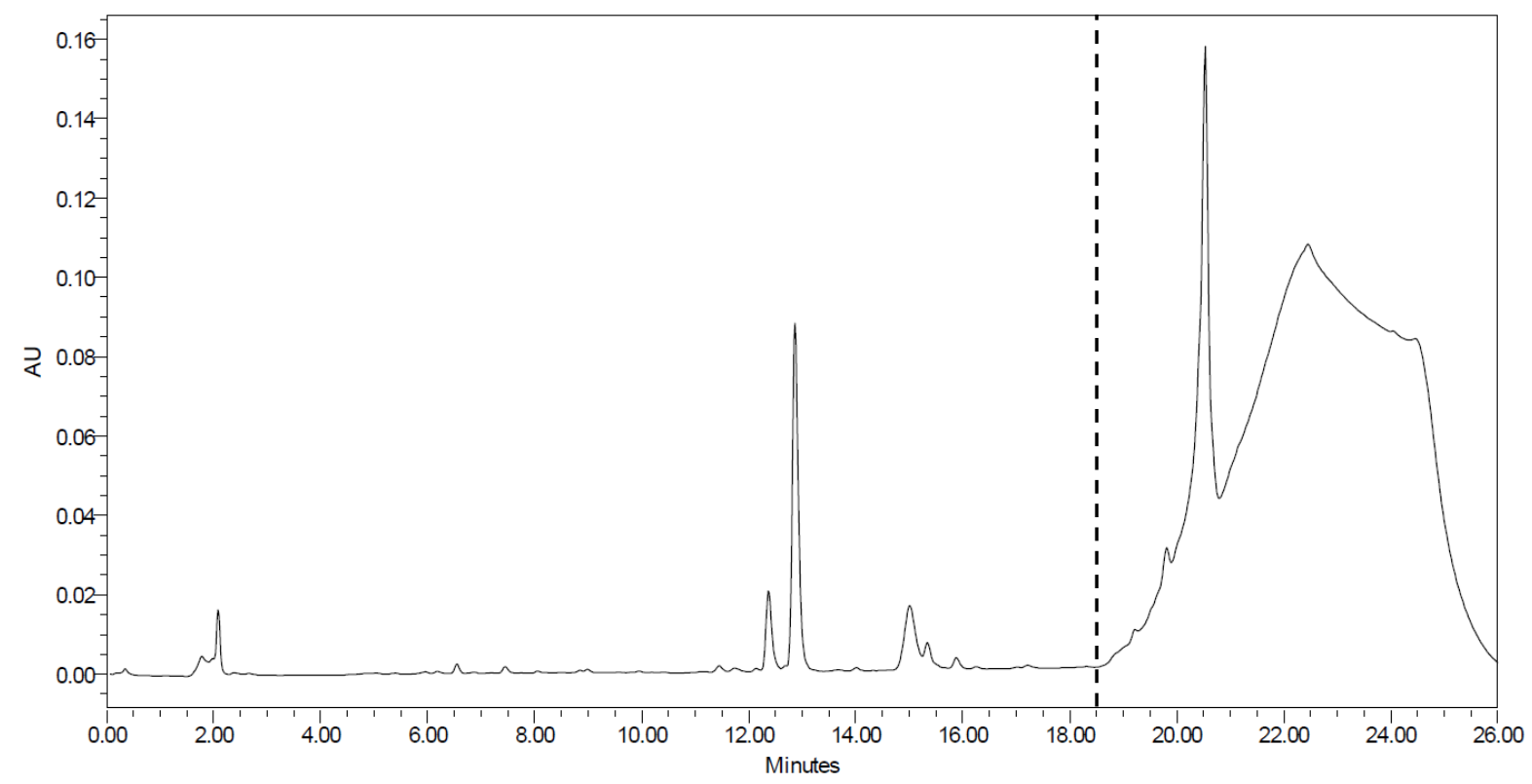

Figure S8. RP-HPLC Chromatogram of crude peptide obtained after coupling of Fmoc-Thr( $t-\mathrm{Bu})$ 3a to peptide 1 with 1.1 MER for one hour in a shaker at $100 \mathrm{rpm}$ (Table 1, Entry 5). 


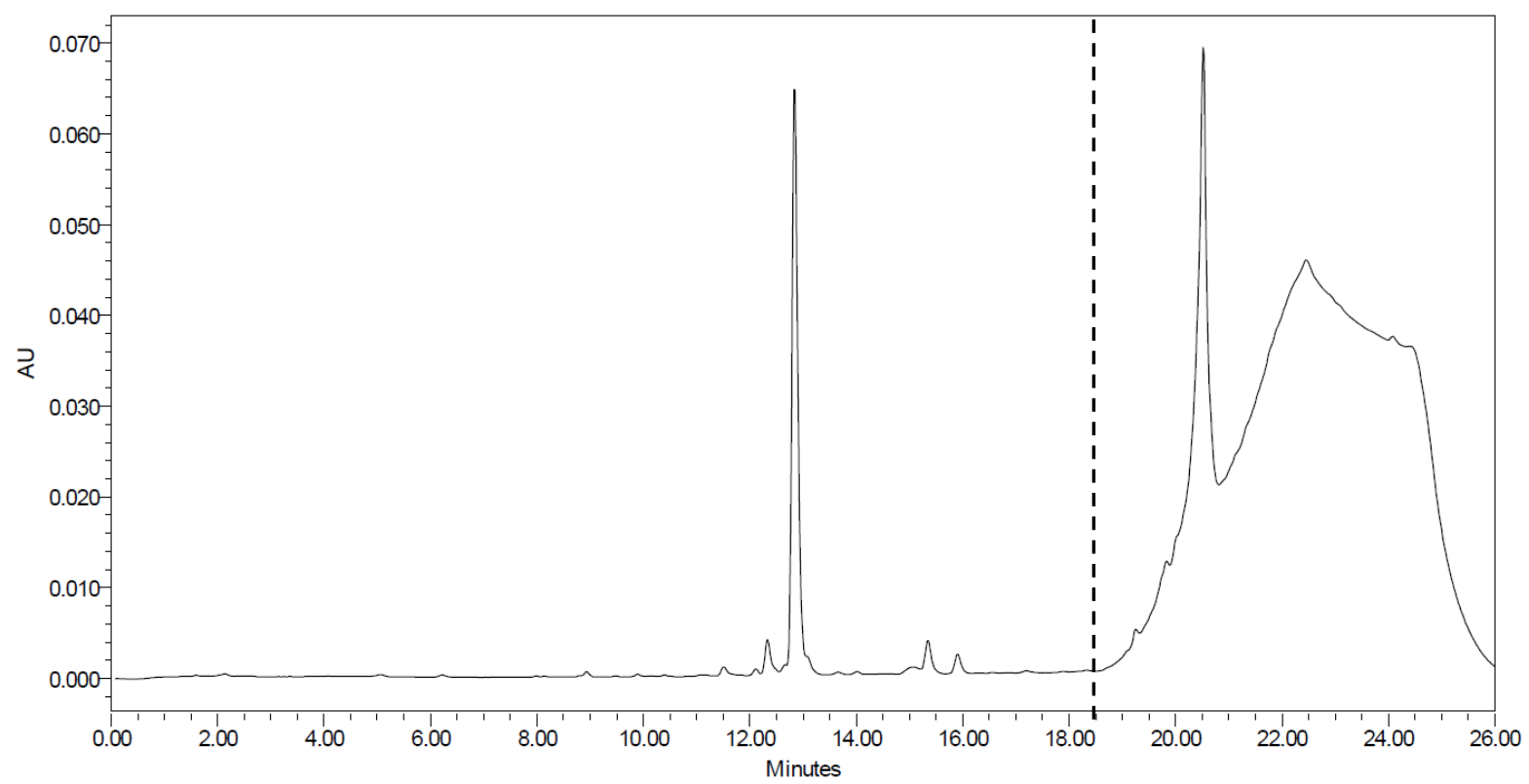

Figure S9. RP-HPLC Chromatogram of crude peptide obtained after coupling of Fmoc-Thr( $t$-Bu) 3a to peptide 1 with 1.5 MER for one hour in a shaker at $100 \mathrm{rpm}$ (Table 1, Entry 7).

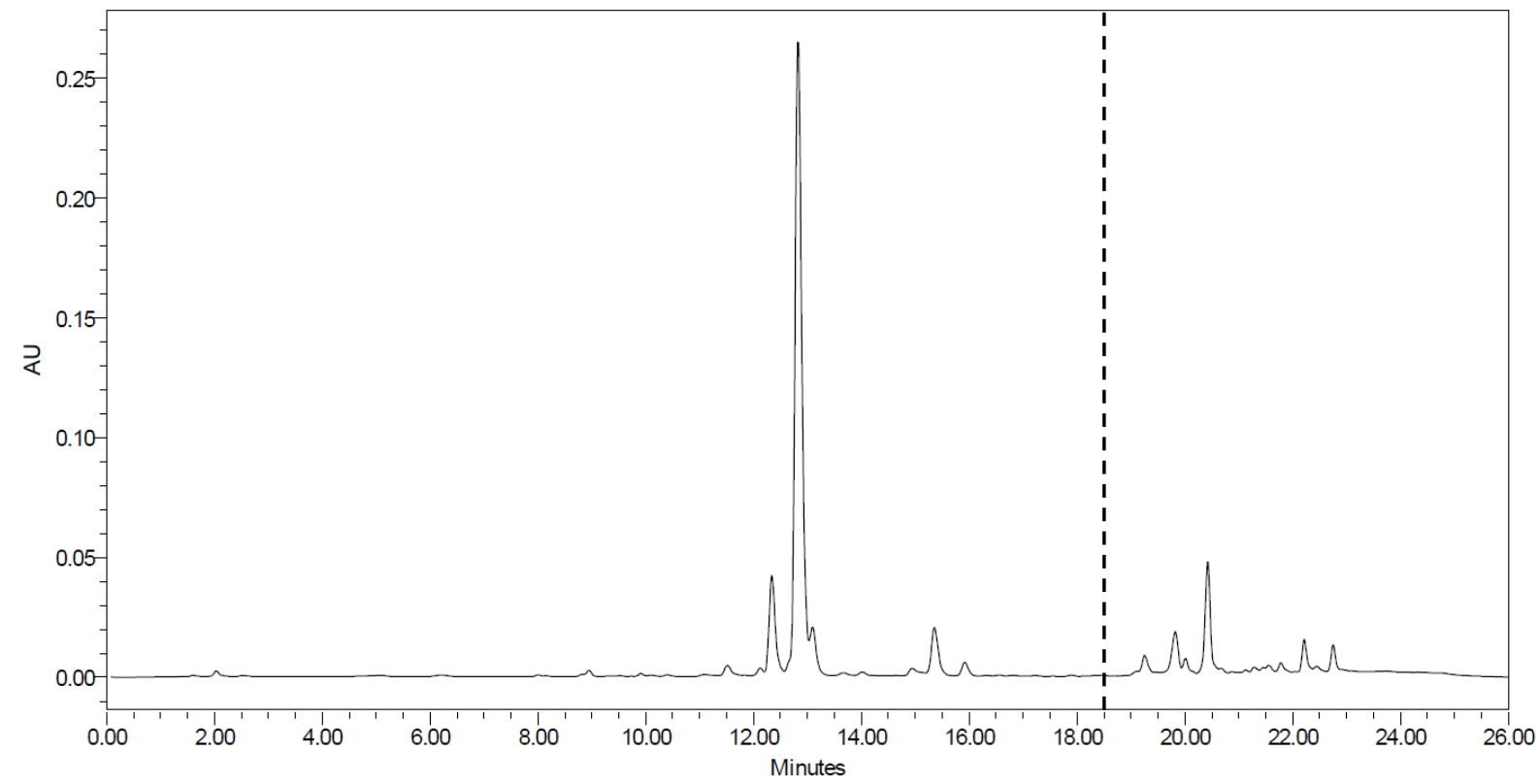

Figure S10. RP-HPLC Chromatogram of crude obtained after coupling of Fmoc-Thr( $t$-Bu) 3a to peptide 1 with 1.1 MER for one hour in HSS at $700 \mathrm{rpm}$ (Table 1, Entry 6). 


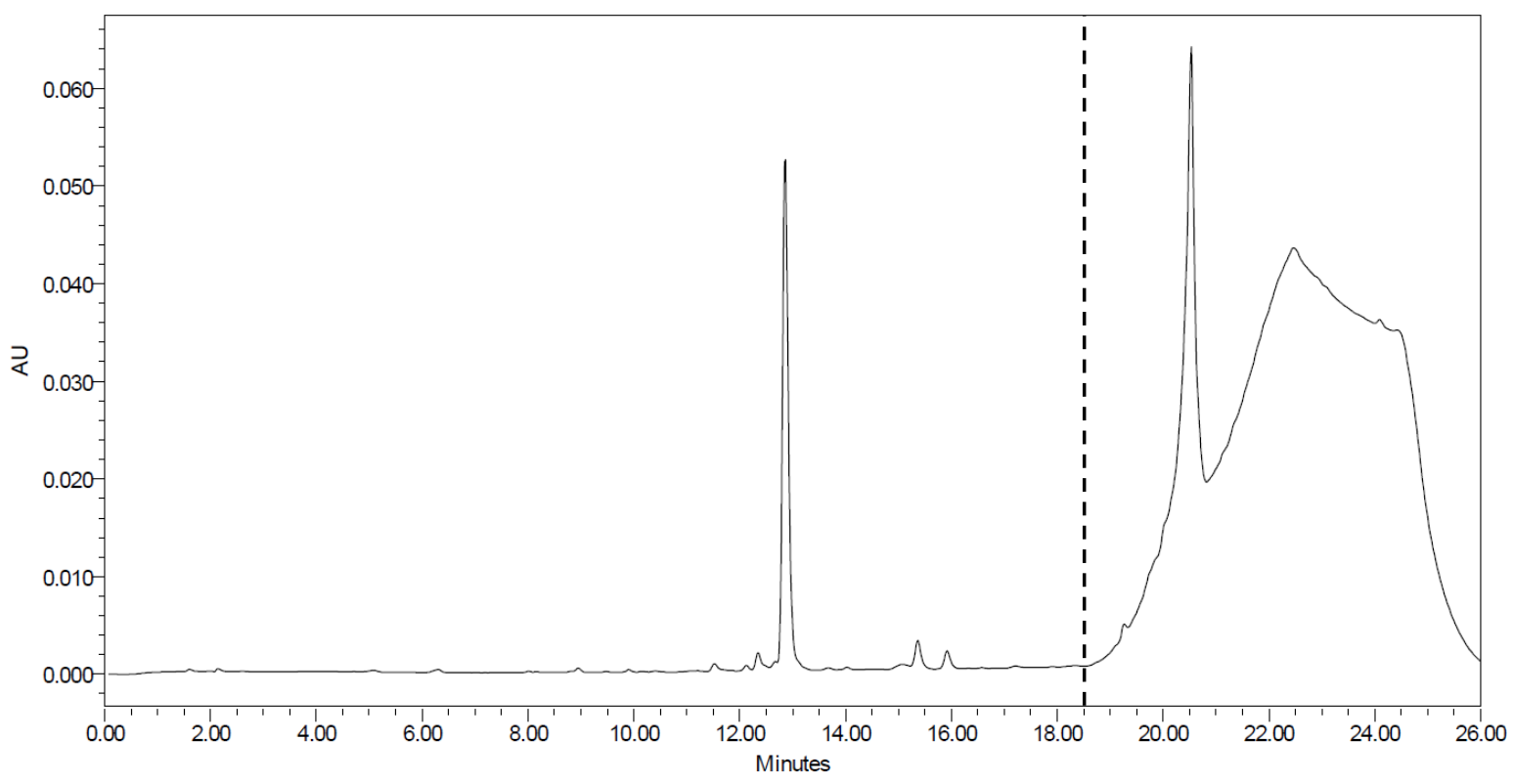

Figure S11. RP-HPLC Chromatogram of crude peptide obtained after coupling of Fmoc-Thr $(t-\mathrm{Bu})$ 3a to peptide 1 with 1.5 MER for one hour in HSS at $700 \mathrm{rpm}$ (Table 1, Entry 8). 


\subsection{HPLC Results of coupling Fmoc-His(Trt) 4a to peptide 1}

Peak at 12.4 minutes corresponds to peptide 1 and peak at 11 minutes corresponds to the product 4. All chromatograms were recorded for crude samples after cleavage without further purification. Peaks after 18 minutes (designated by the vertical dashed line) are related to residual protecting group moieties and to the HPLC gradient program, which corresponds to washing region and do not contain the peptides that are relevant for this study.

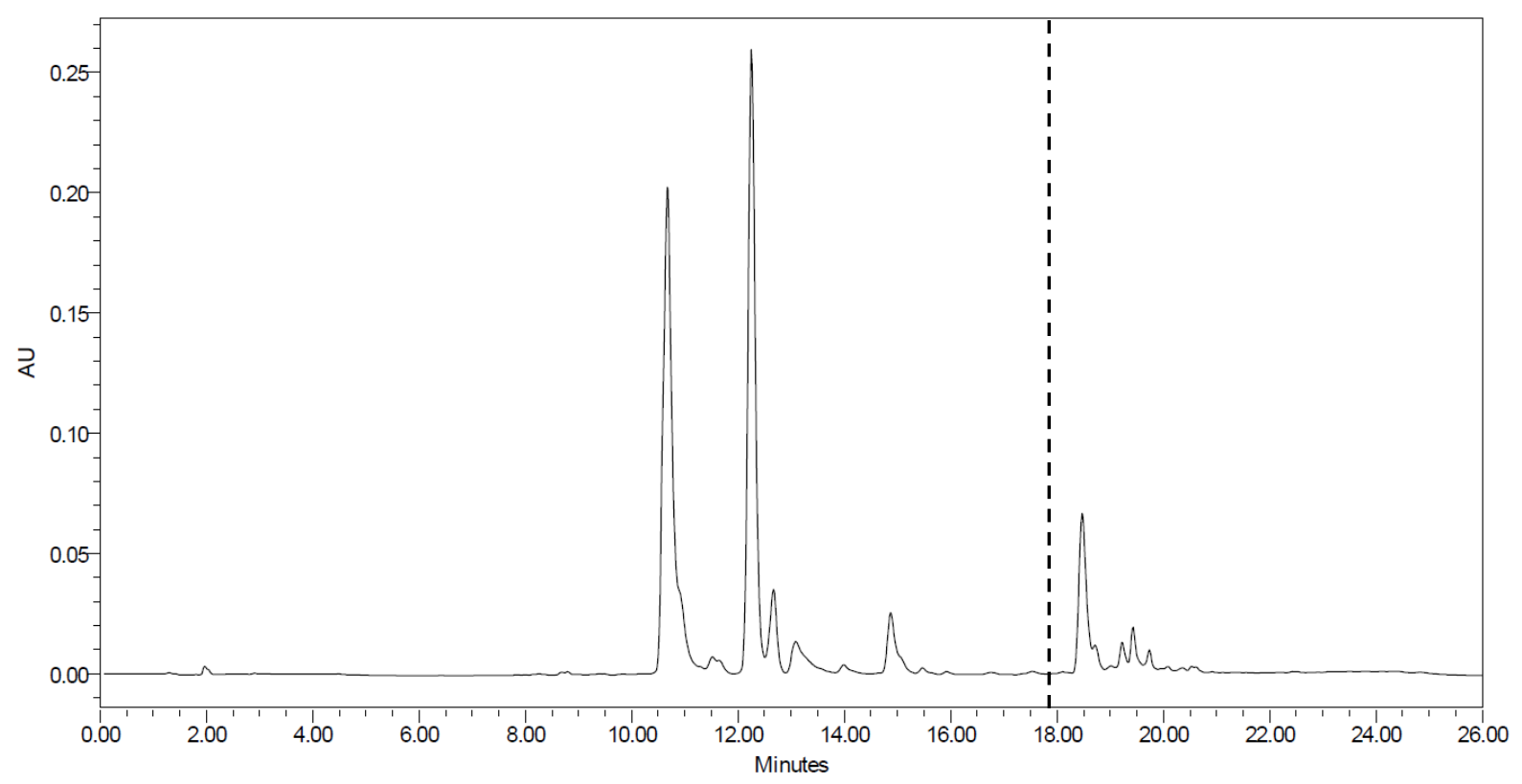

Figure S12. RP-HPLC Chromatogram of crude obtained after coupling of Fmoc-His(Trt) 4a to peptide 1 with 1.1 MER for one hour in a shaker at $100 \mathrm{rpm}$ (Table 1, Entry 9). 


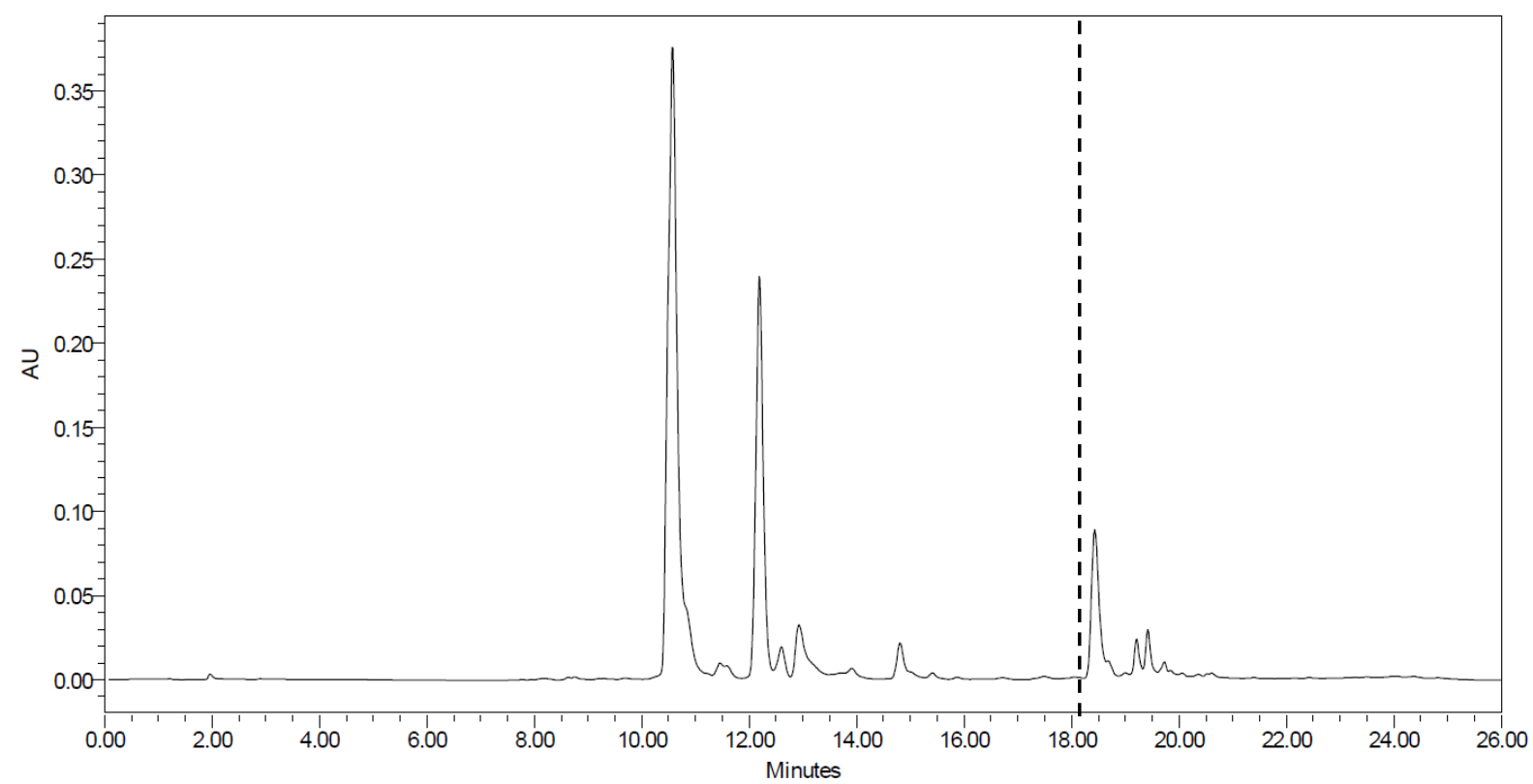

Figure S13. RP-HPLC Chromatogram of crude obtained after coupling of Fmoc-His(Trt) 4a to peptide 1 with 1.5 MER for one hour in a shaker at $100 \mathrm{rpm}$ (Table 1, Entry 11).

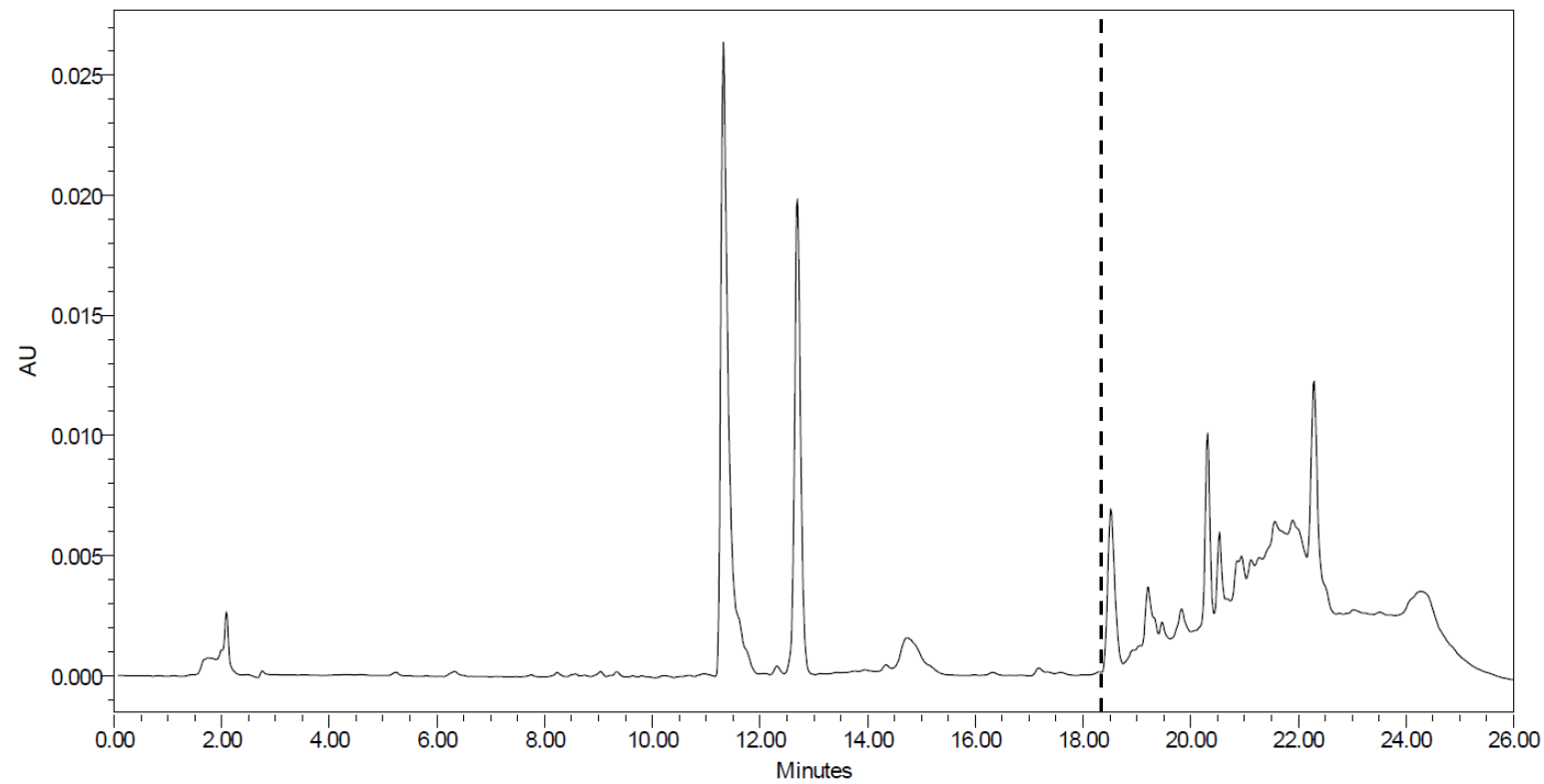

Figure S14. RP-HPLC Chromatogram of crude peptide obtained after coupling of Fmoc-His(Trt) 4a to peptide 1 with 1.1 MER for one hour in HSS at $700 \mathrm{rpm}$ (Table 1, Entry 10). 


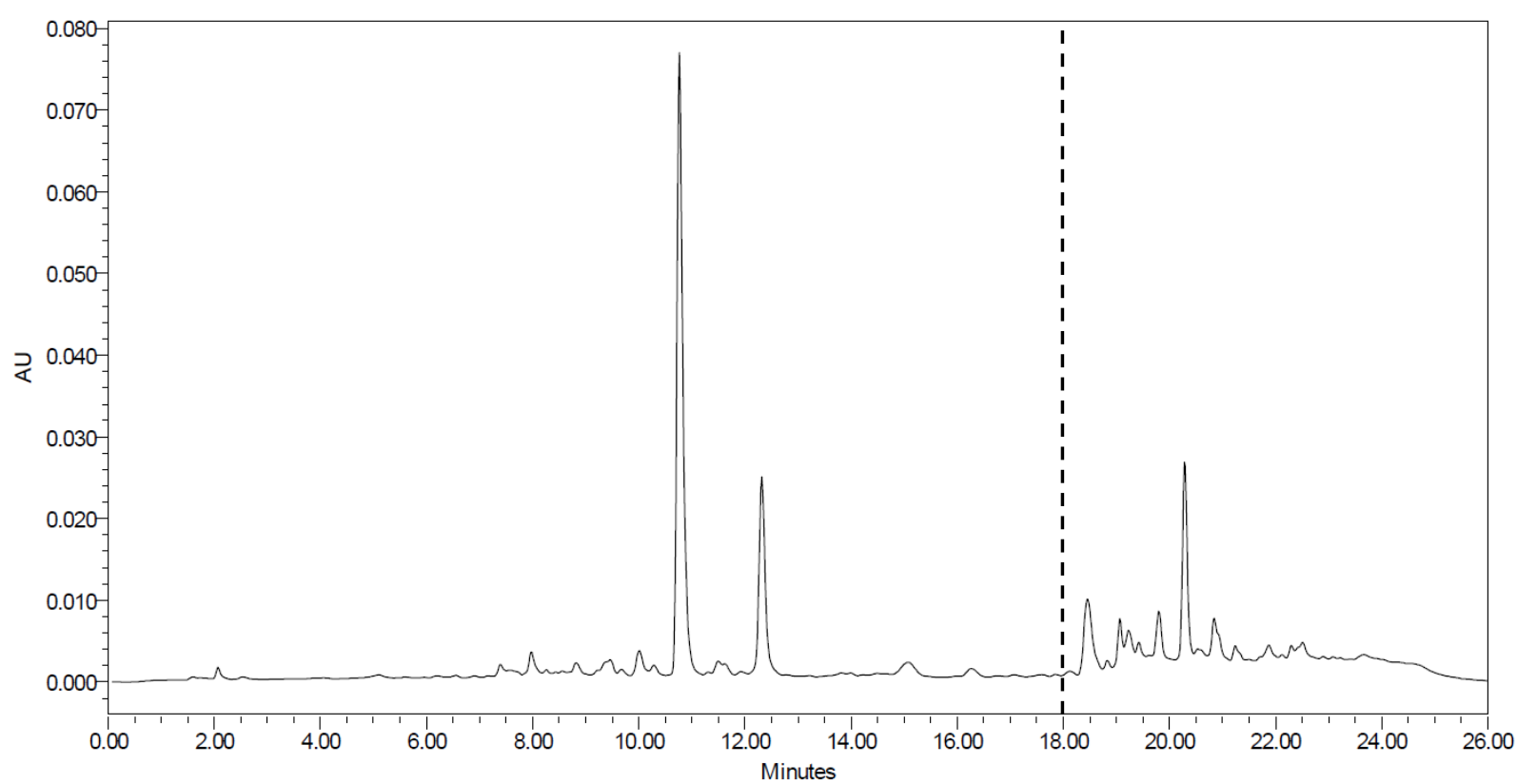

Figure S15. RP-HPLC Chromatogram of crude obtained after coupling of Fmoc-His(Trt) 4a to peptide 1 with 1.5 MER for one hour in HSS at $700 \mathrm{rpm}$ (Table 1, Entry 12). 


\subsection{HPLC Results of stirring effect}

Peak at 12.5 minutes corresponds to peptide 1 and peak at 11 minutes corresponds to the product 4. All chromatograms were recorded for crude samples after cleavage without further purification. Peaks after 18 minutes (designated by the vertical dashed line) are related to residual protecting group moieties and to the HPLC gradient program, which corresponds to washing region and do not contain the peptides that are relevant for this study.

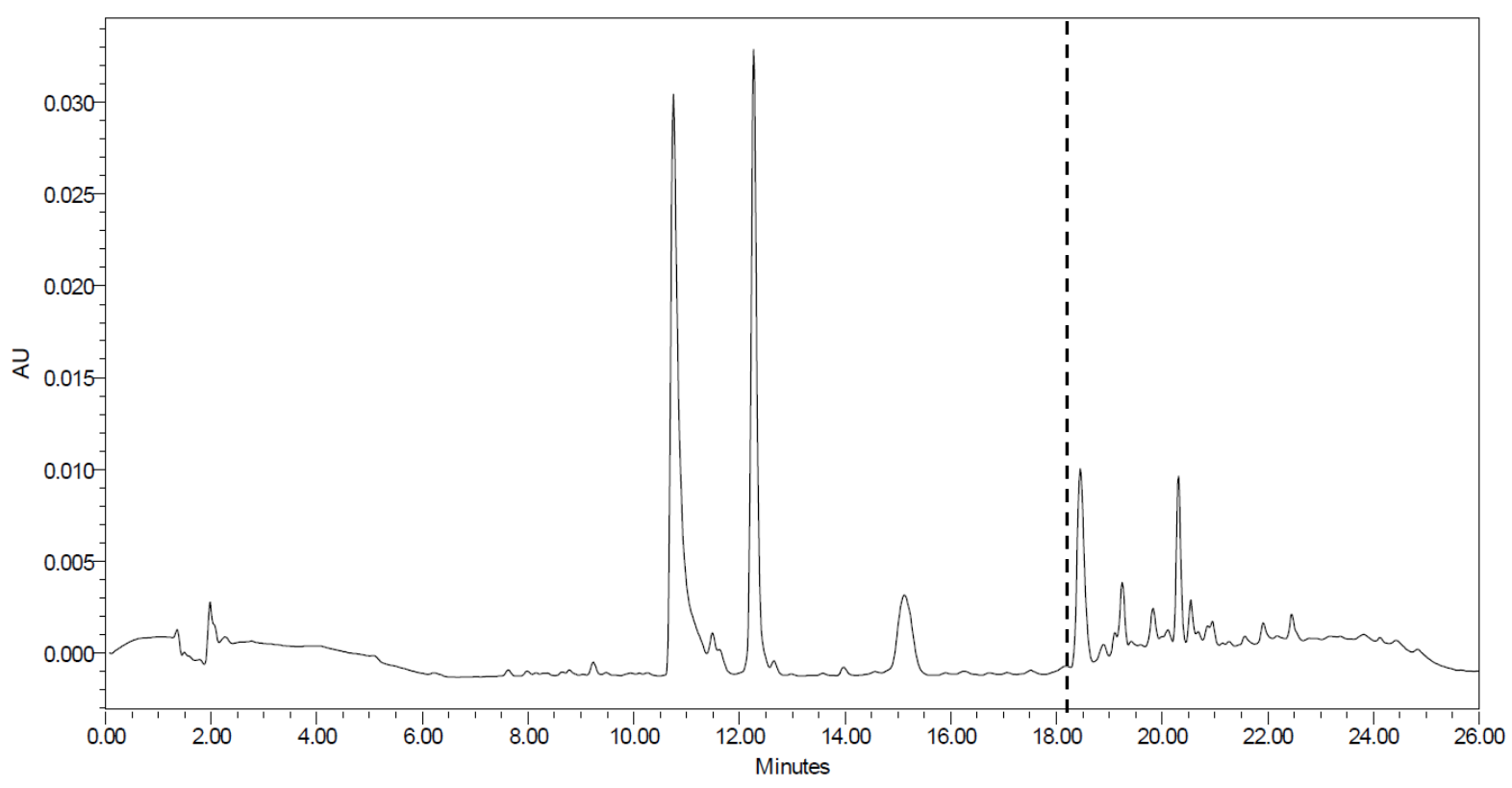

Figure S16. RP-HPLC Chromatogram of crude obtained after coupling of Fmoc-His(Trt) 4a to peptide 1 with 1.1 MER for one hour in HSS at $100 \mathrm{rpm}$. 


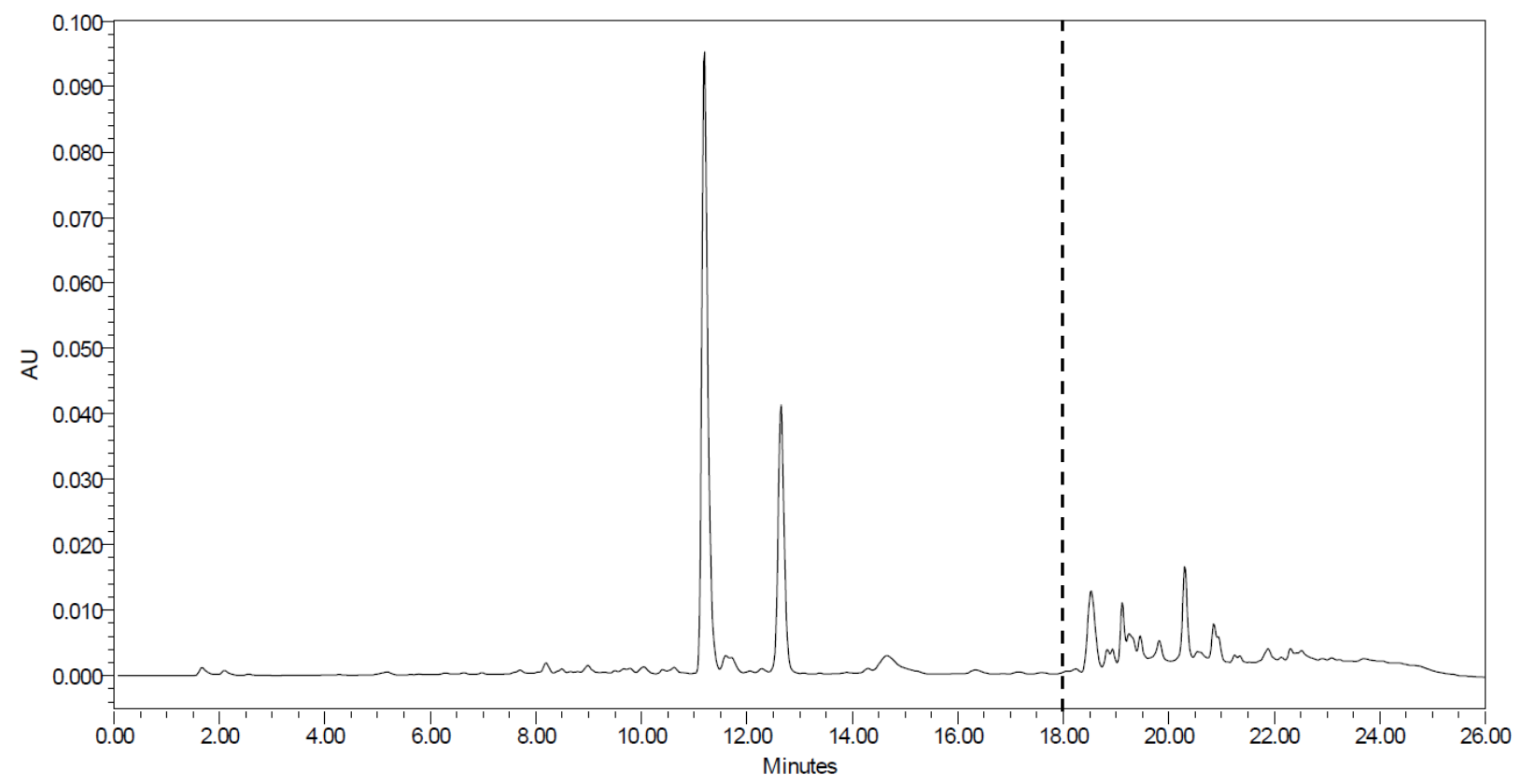

Figure S17. RP-HPLC Chromatogram of crude obtained after coupling of Fmoc-His(Trt) 4a to peptide 1 with 1.1 MER for one hour in HSS at $900 \mathrm{rpm}$. 


\subsection{HPLC Results of Fmoc-His(Trt) 4a preactivation}

Peak at 12.5 minutes corresponds to peptide 1 and peak at 11 minutes corresponds to the product 4. All chromatograms were recorded for crude samples after cleavage without further purification. Peaks after 18 minutes (designated by the vertical dashed line) are related to residual protecting group moieties and to the HPLC gradient program, which corresponds to washing region and do not contain the peptides that are relevant for this study.

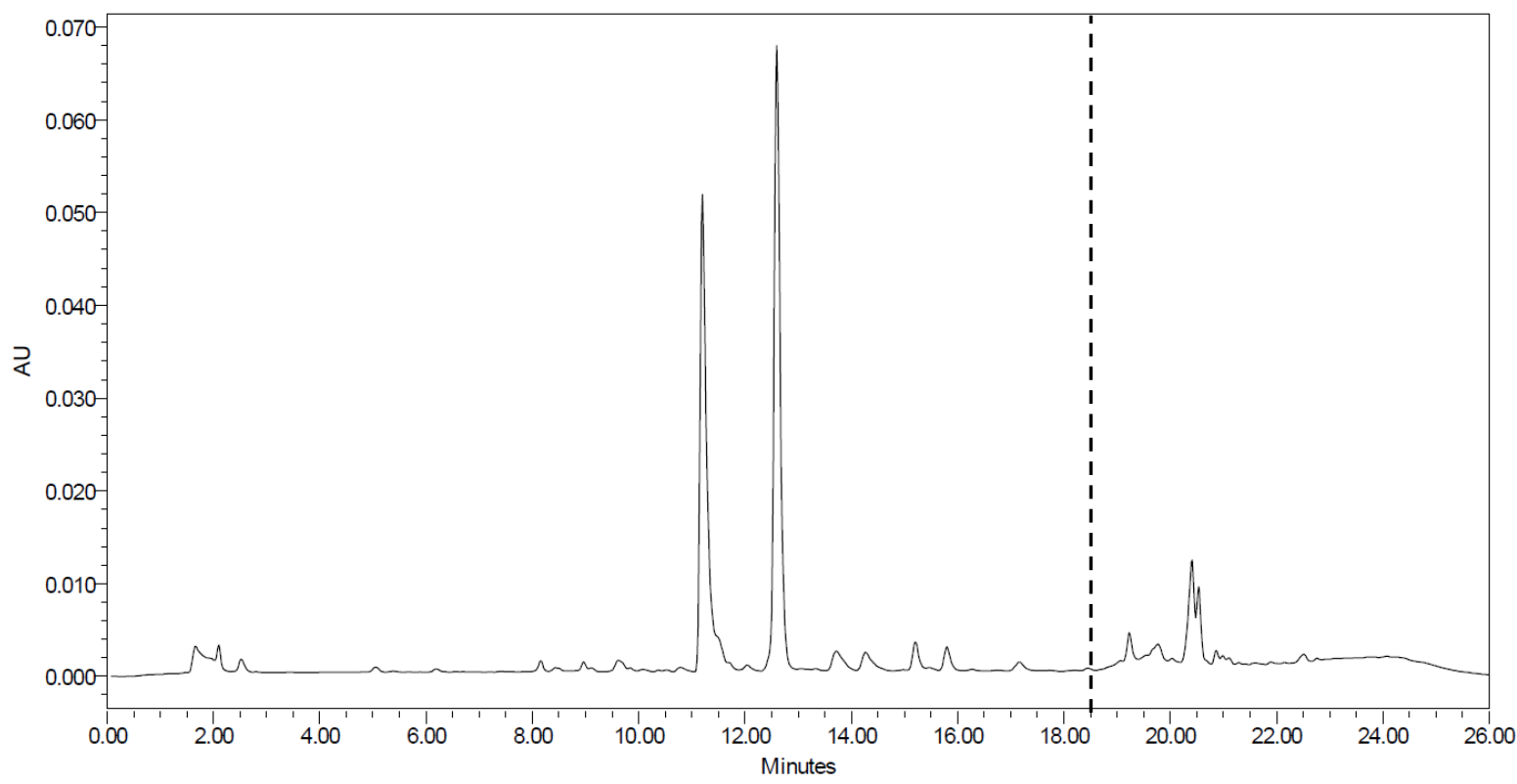

Figure S18. RP-HPLC Chromatogram of crude obtained after coupling of Fmoc-His(Trt) 4a to peptide 1 with 1.1 MER for one hour in a shaker at $100 \mathrm{rpm}$ without preactivation. 


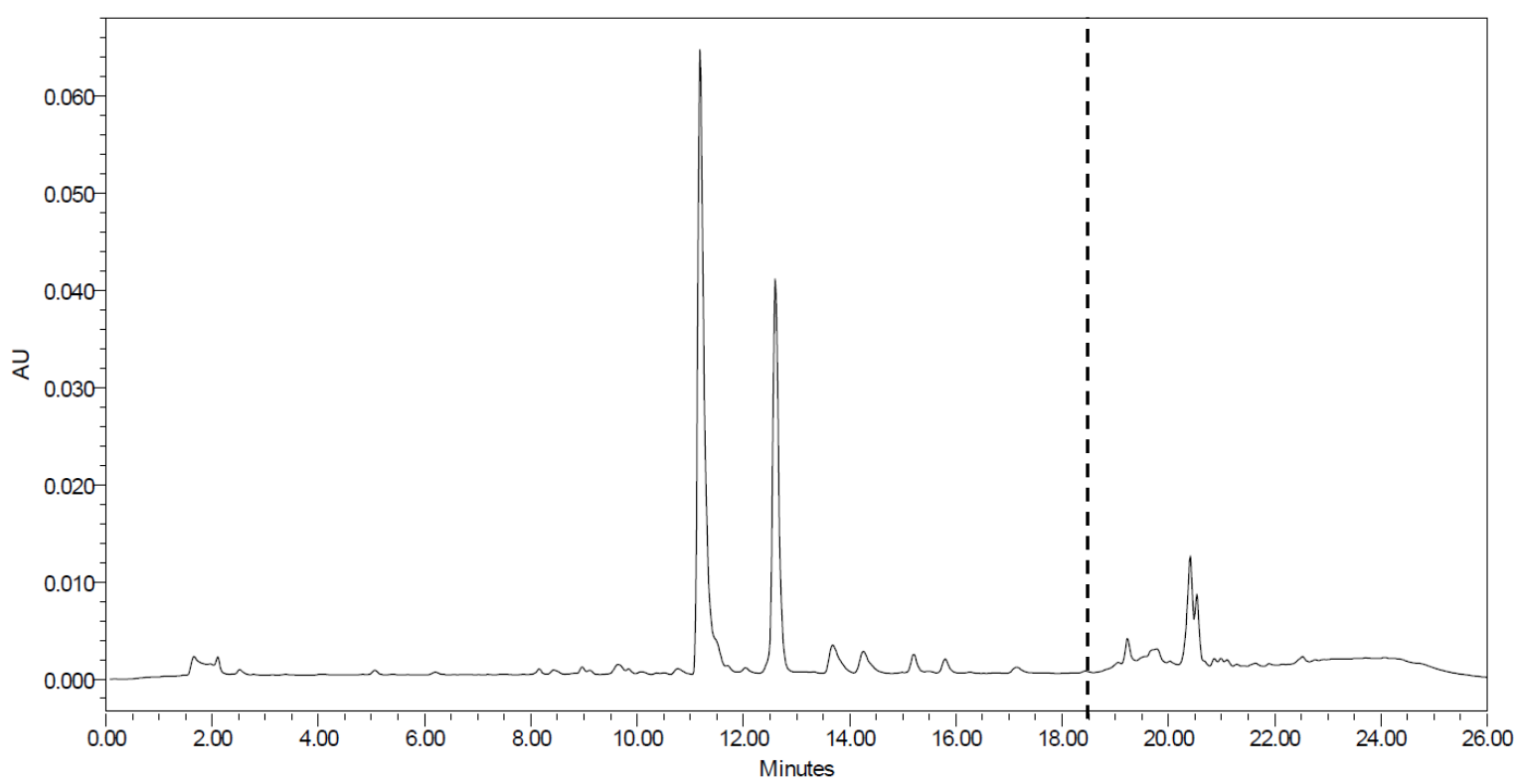

Figure S19. RP-HPLC Chromatogram of crude obtained after coupling of Fmoc-His(Trt) 4a to peptide 1 with 1.1 MER for two hours in HSS at $700 \mathrm{rpm}$ with preactivation. 


\subsection{HPLC Result for the coupling time duration studies}

Peak at 12.5 minutes corresponds to peptide 1 and peak at 11 minutes corresponds to the product 4. All chromatograms were recorded for crude samples after cleavage without further purification. Peaks after 18 minutes (designated by the vertical dashed line) are related to residual protecting group moieties and to the HPLC gradient program, which corresponds to washing region and do not contain the peptides that are relevant for this study.

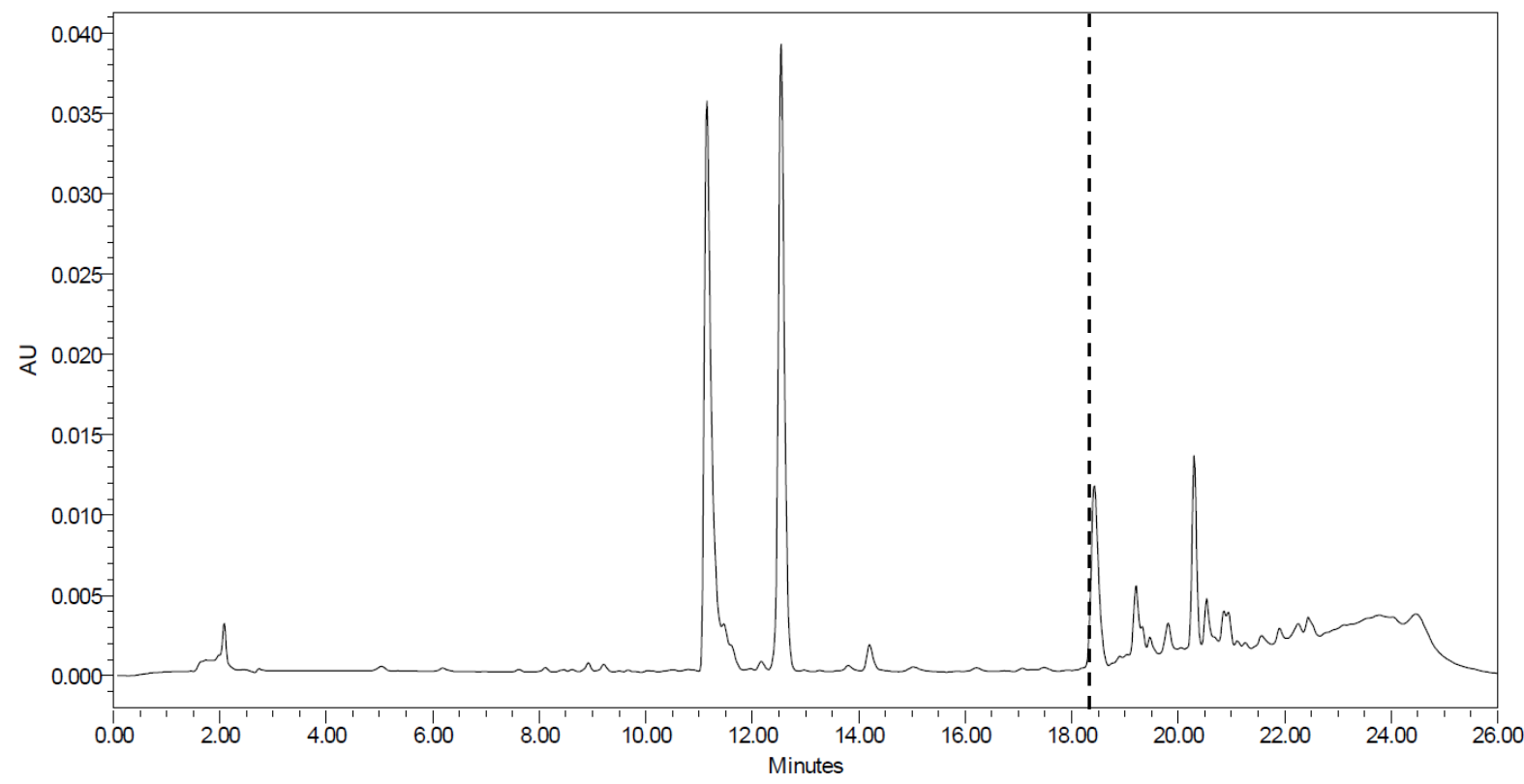

Figure S20. RP-HPLC Chromatogram of the crude obtained after coupling of Fmoc-His(Trt) 4a to peptide 1 with 1.1 MER overnight (16 hours) in a shaker at $100 \mathrm{rpm}$. 


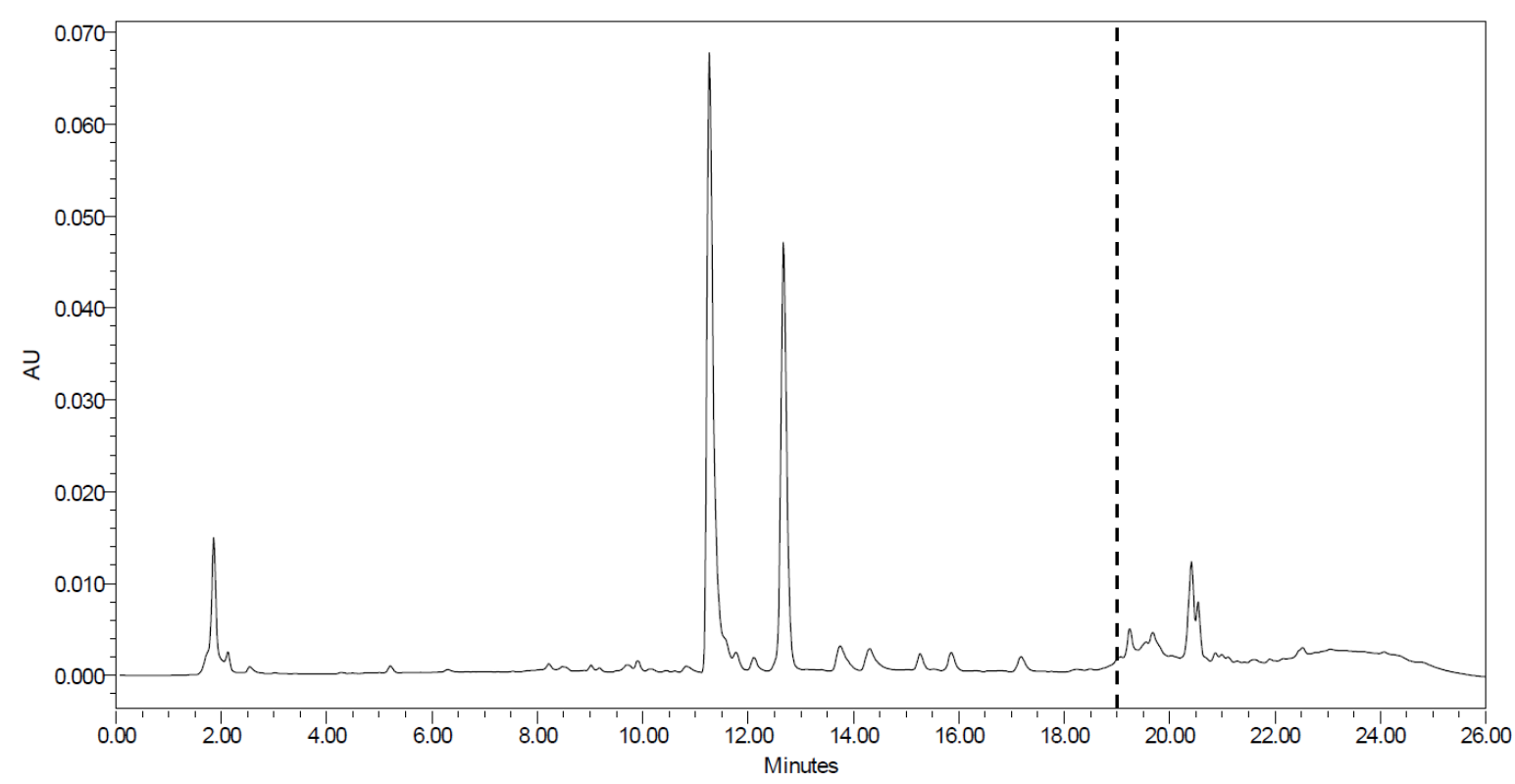

Figure S21. RP-HPLC Chromatogram of the crude obtained after coupling of Fmoc-His(Trt) 4a to peptide 1 with 1.1 MER for two hours in HSS at $700 \mathrm{rpm}$. 
3.9 HPLC Results for the effect of duration during preactivation with HATU as coupling agent

Peak at 12.5 minutes corresponds to peptide $\mathbf{1}$ and peak at 13 minutes corresponds to the product 2. All chromatograms were recorded for crude samples after cleavage without further purification. Peaks after 18 minutes (designated by the vertical dashed line) are related to residual protecting group moieties and to the HPLC gradient program, which corresponds to washing region and do not contain the peptides that are relevant for this study.

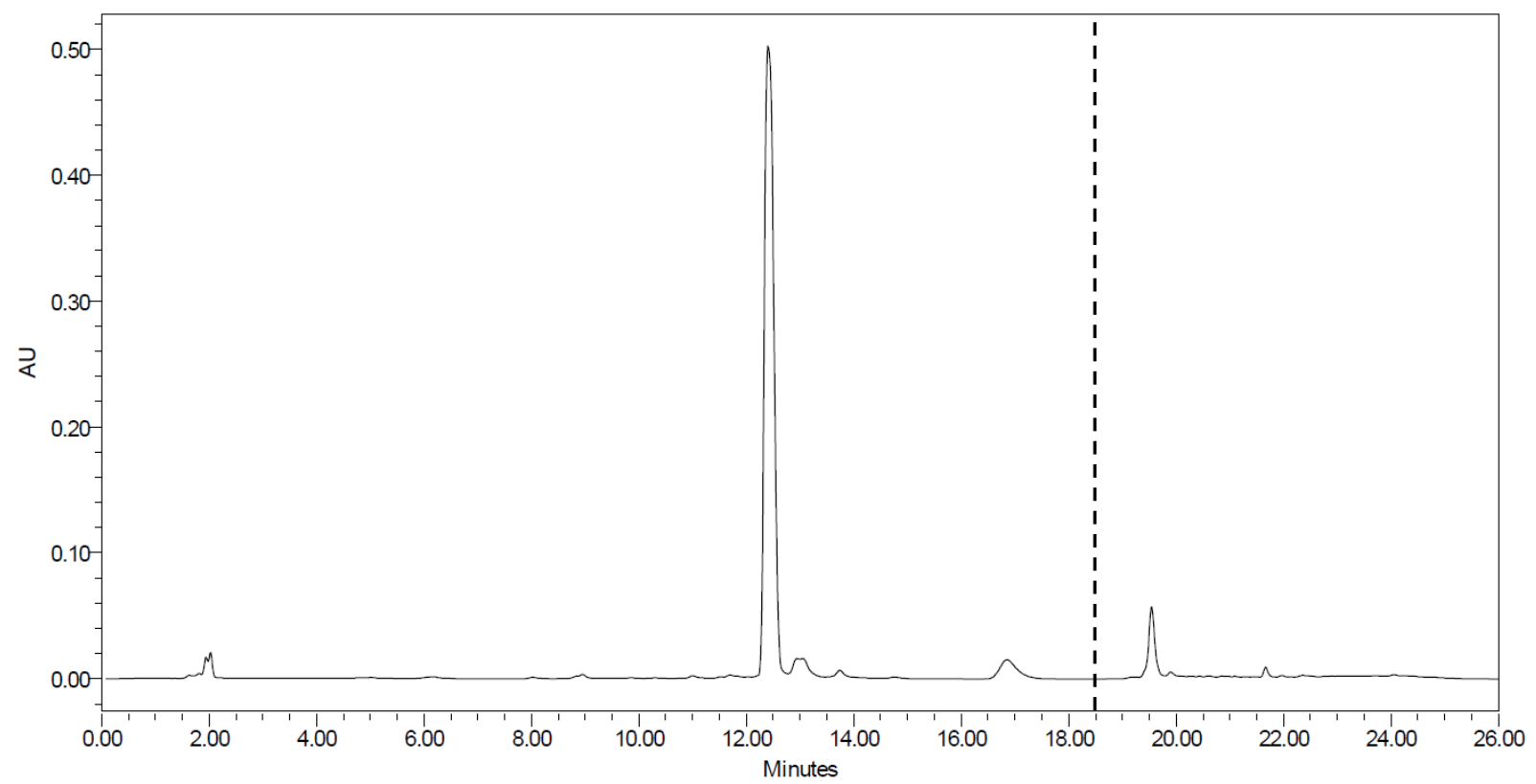

Figure S22. RP-HPLC Chromatogram of the crude obtained after coupling with preactivation of $1 \mathrm{~min}$. The peak at 12.5 was shifted and confirmed by MS to correspond to product $\mathbf{2}$. 


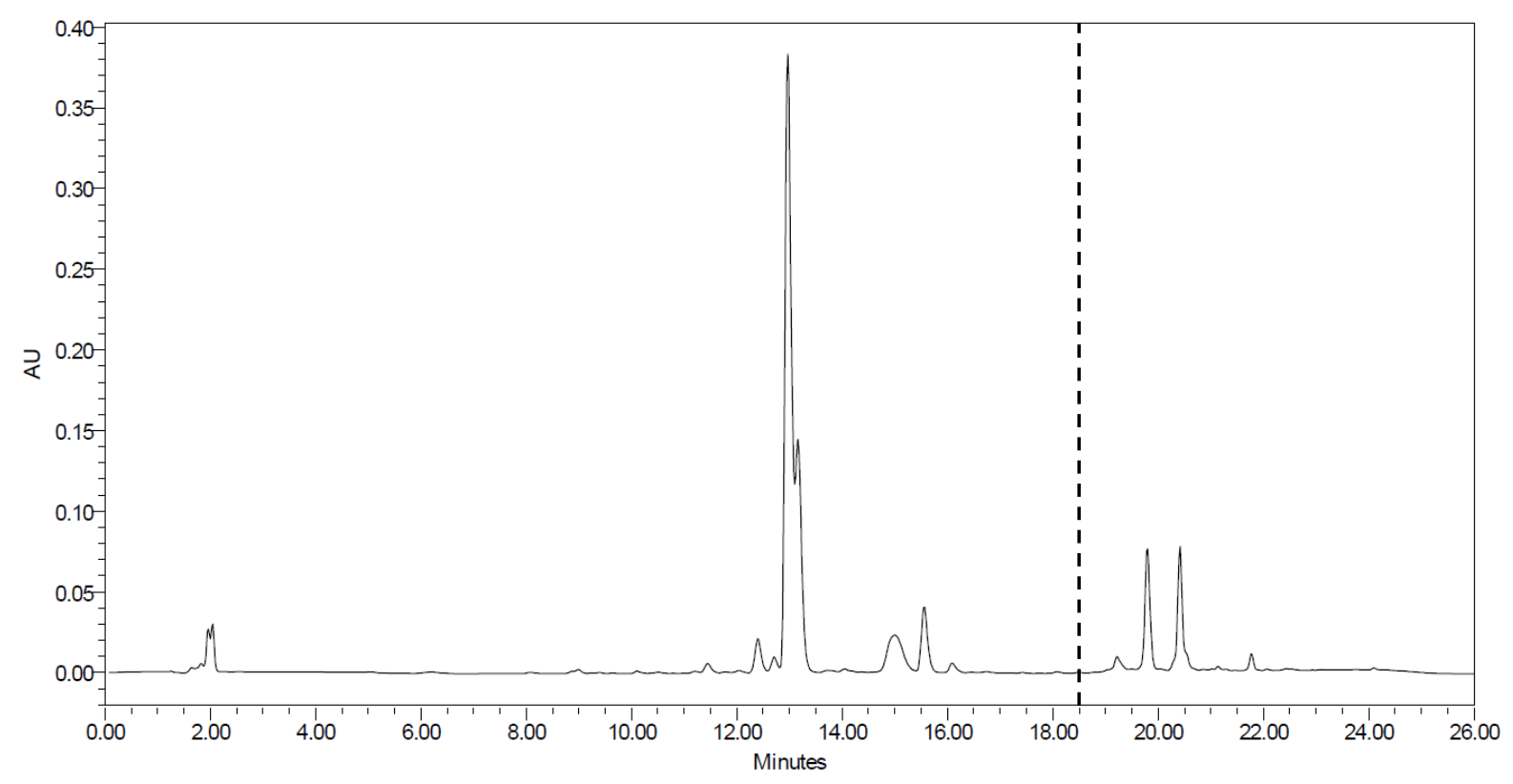

Figure S23. RP-HPLC Chromatogram of the crude obtained after coupling with preactivation of 5 min.

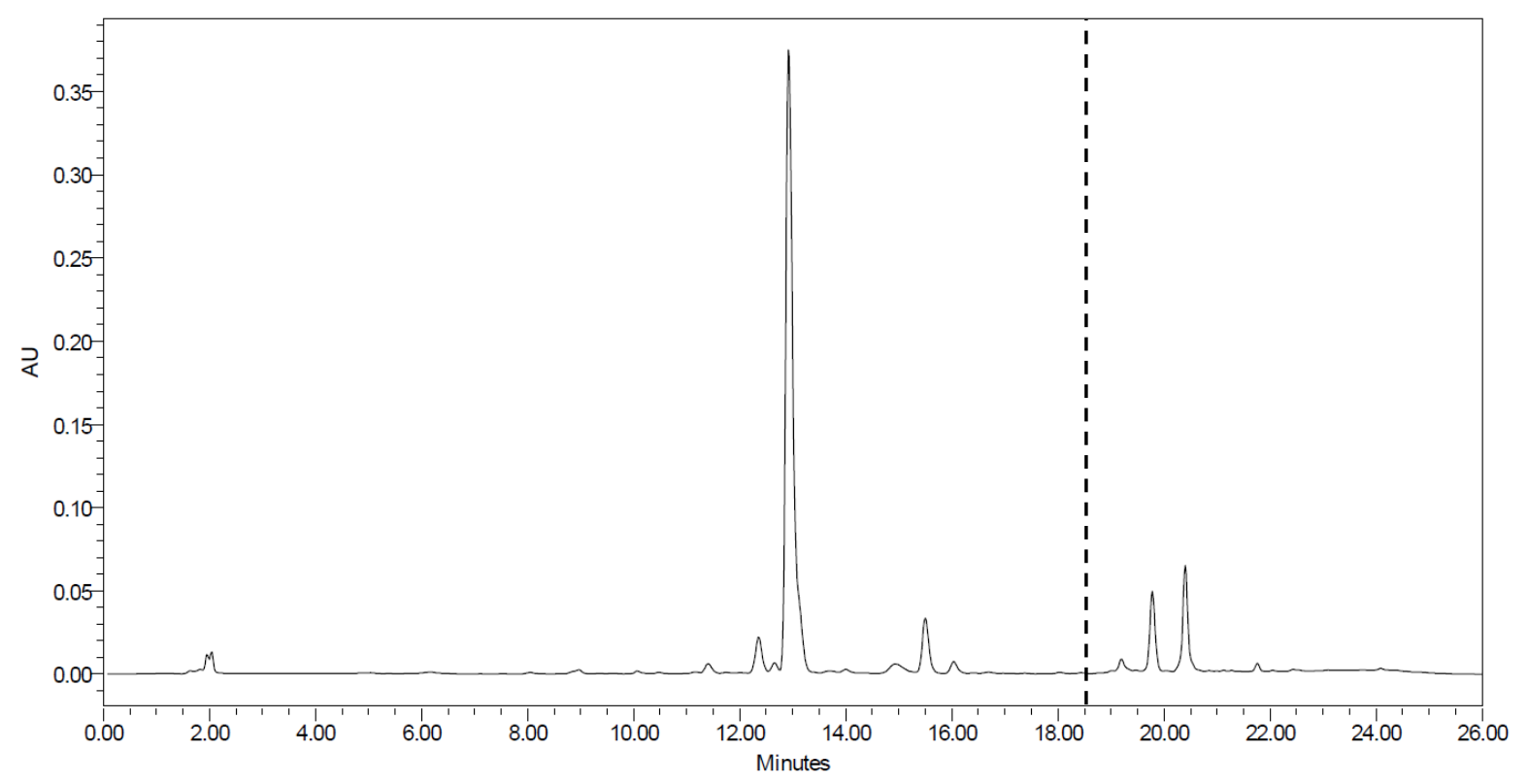

Figure S24. RP-HPLC Chromatogram of the crude obtained after coupling with preactivation of $10 \mathrm{~min}$. 


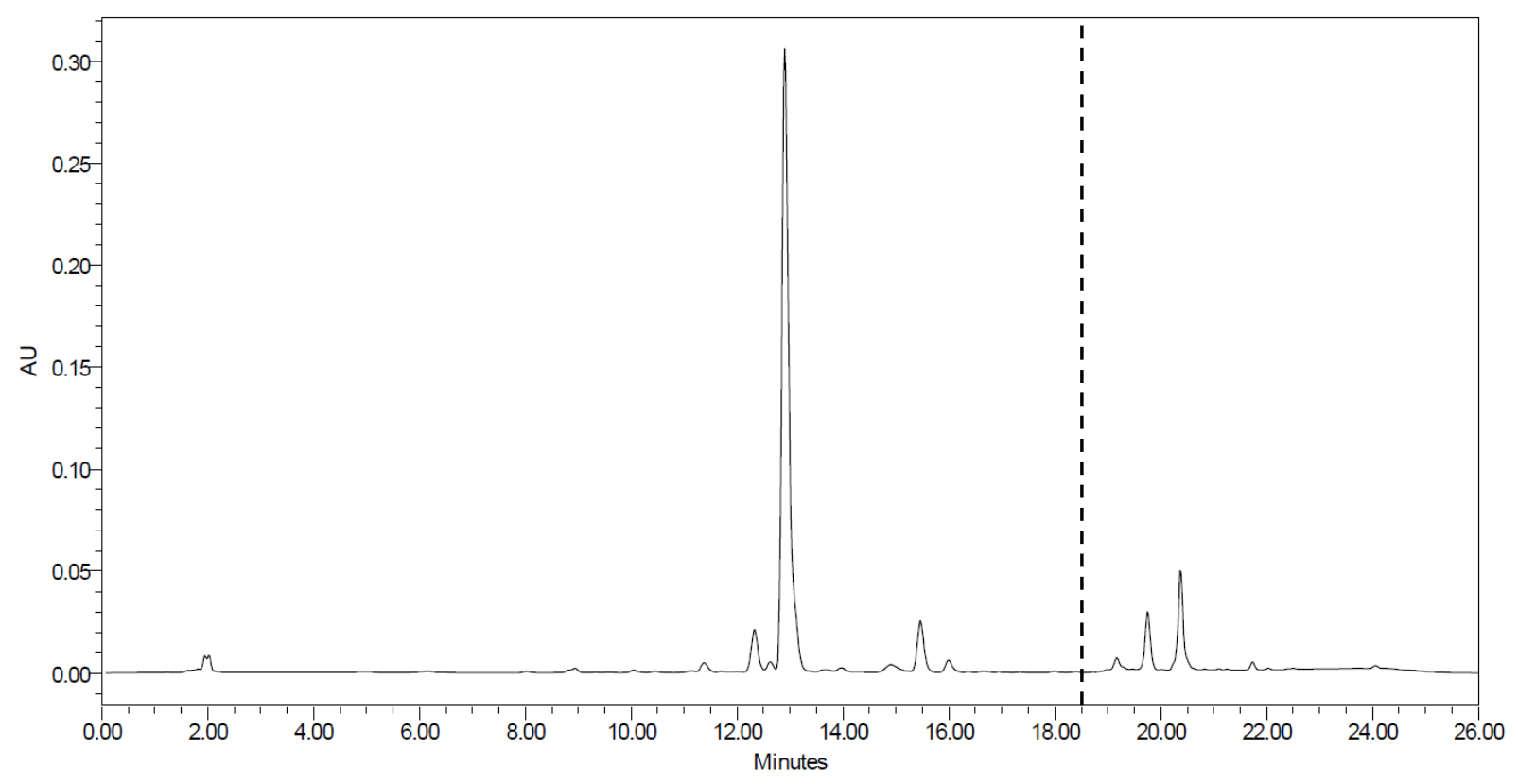

Figure S25. RP-HPLC Chromatogram of the crude obtained after coupling with preactivation of $20 \mathrm{~min}$.

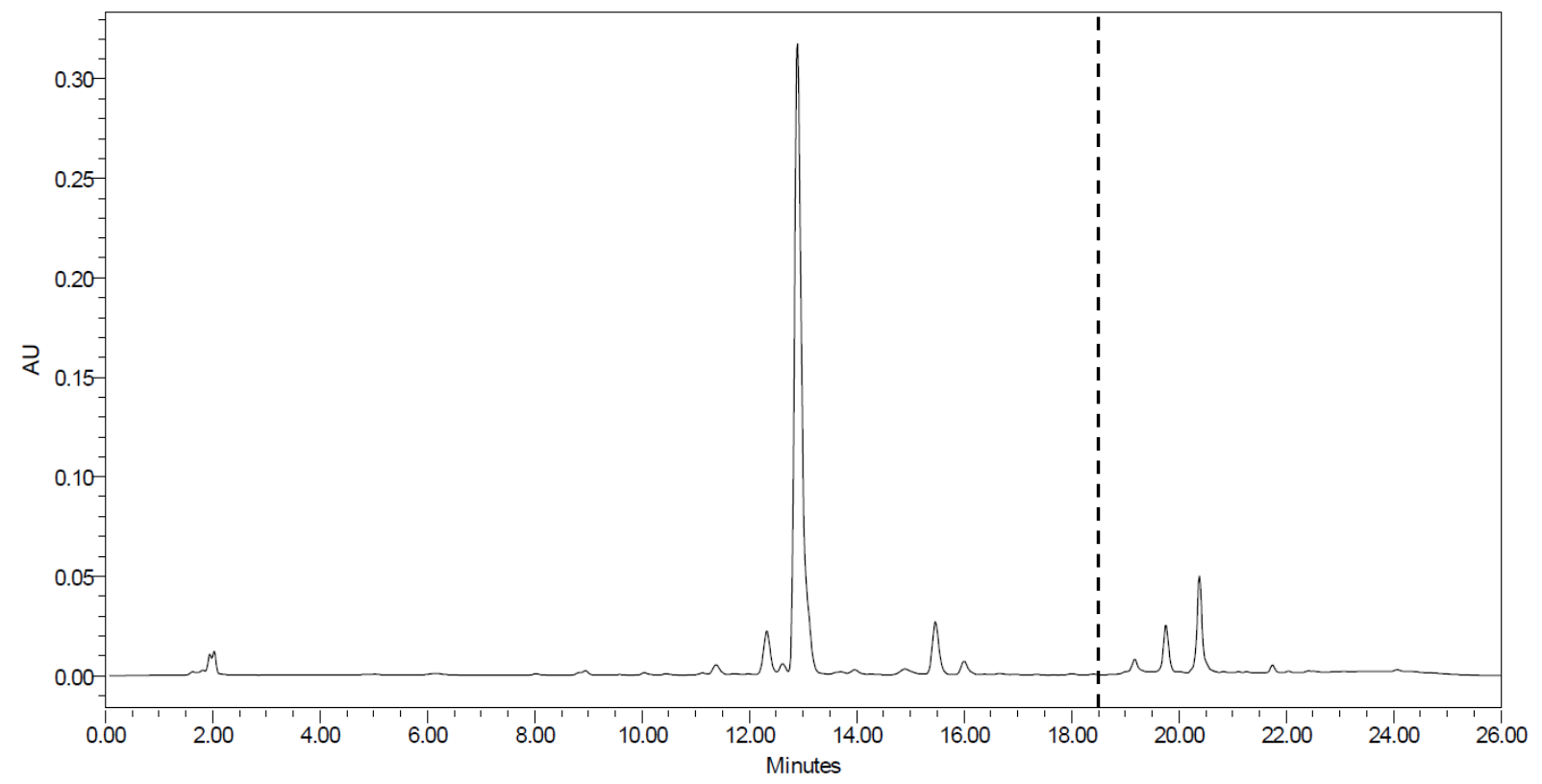

Figure S26. RP-HPLC Chromatogram of the crude obtained after coupling with preactivation of $40 \mathrm{~min}$. 


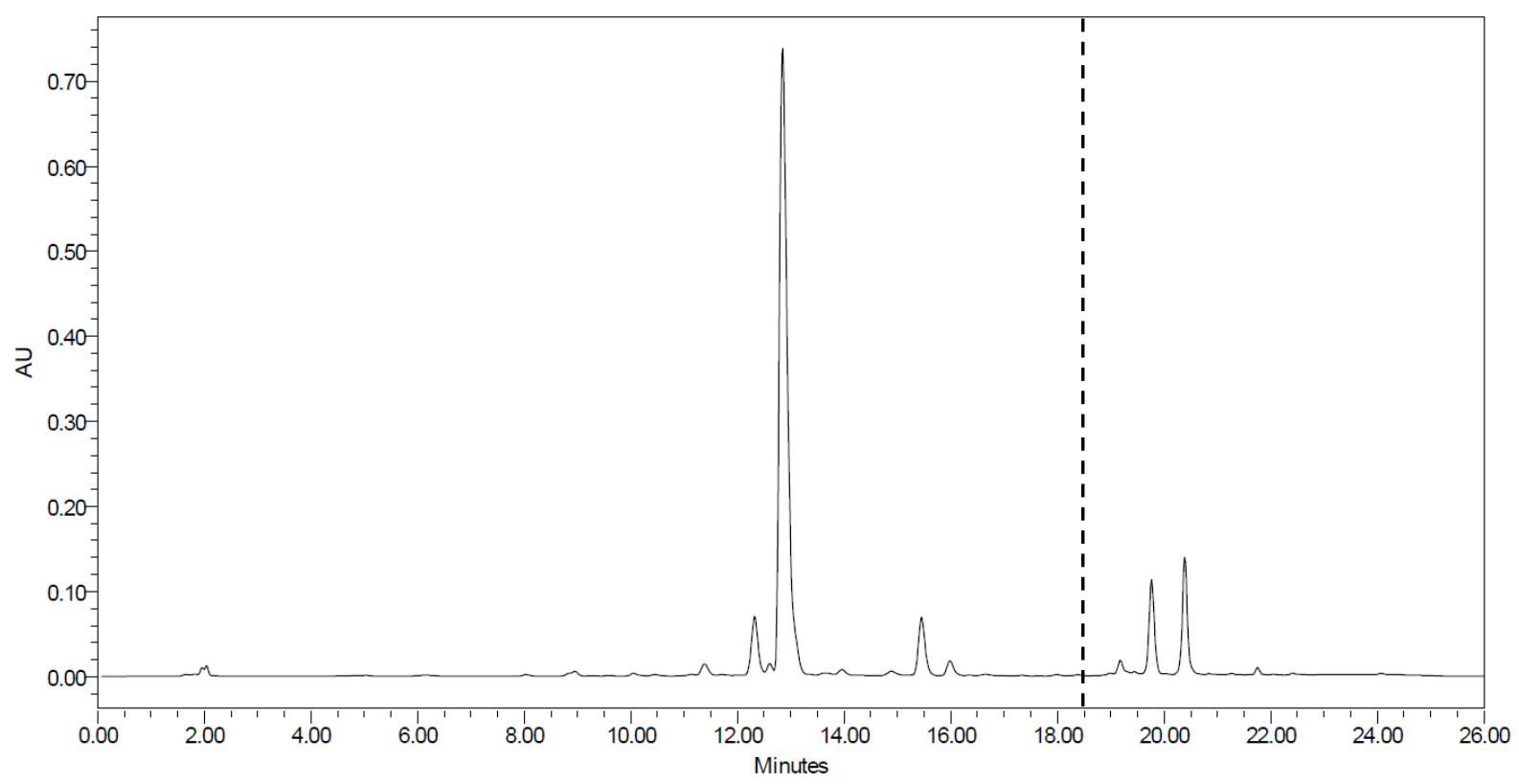

Figure S27. RP-HPLC Chromatogram of crude obtained after coupling with preactivation of 60 min. 
3.10 HPLC Results for the effect of duration during preactivation with HBTU as coupling agent

Peak at 12.5 minutes corresponds to peptide $\mathbf{1}$ and peak at 13 minutes corresponds to the product 2. All chromatograms were recorded for crude samples after cleavage without further purification. Peaks after 18 minutes (designated by the vertical dashed line) are related to residual protecting group moieties and the HPLC gradient program which corresponds to washing region and do not contain the peptides that are relevant for this study.

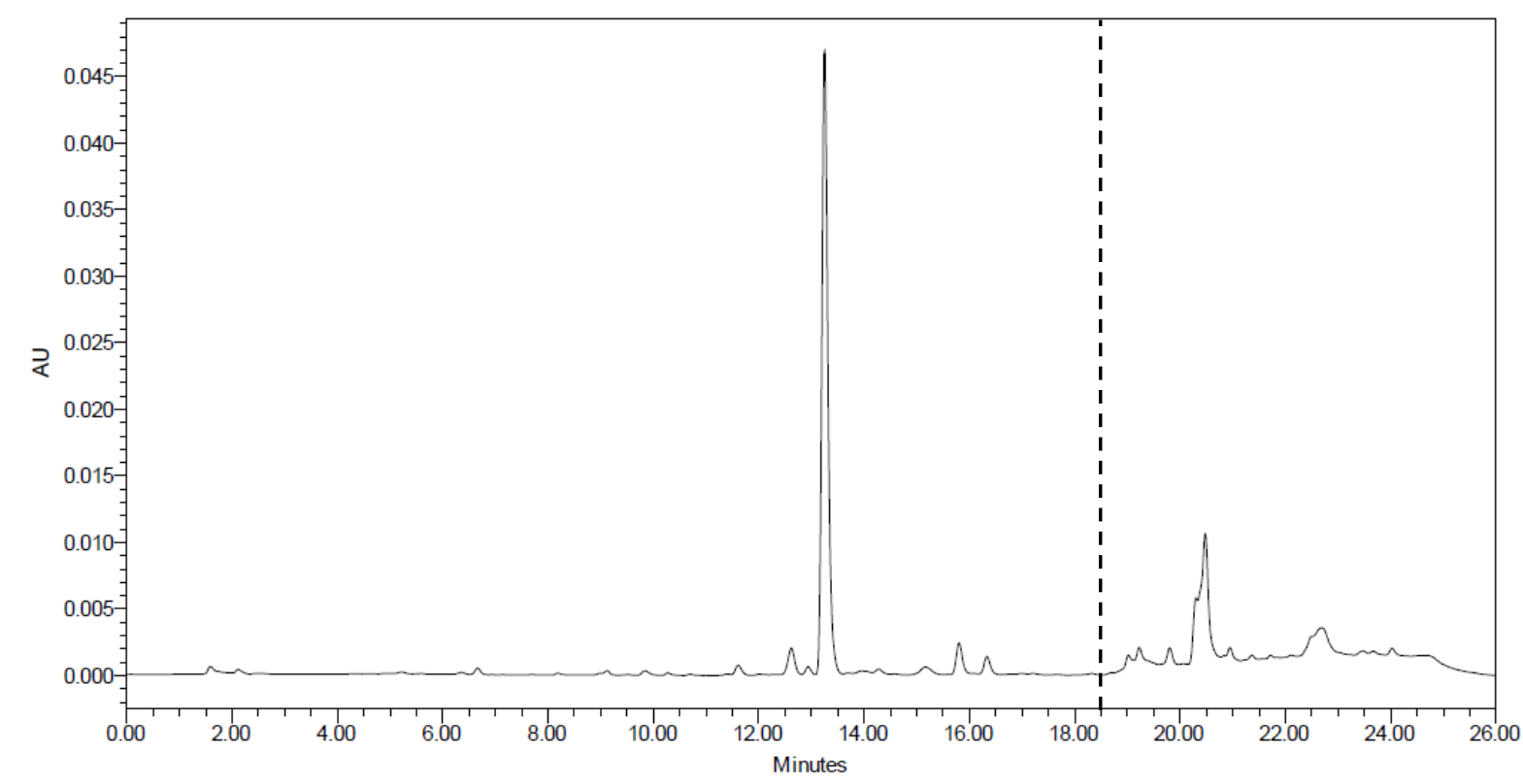

Figure S28. RP-HPLC Chromatogram of the crude obtained after coupling with preactivation of 1 min. 


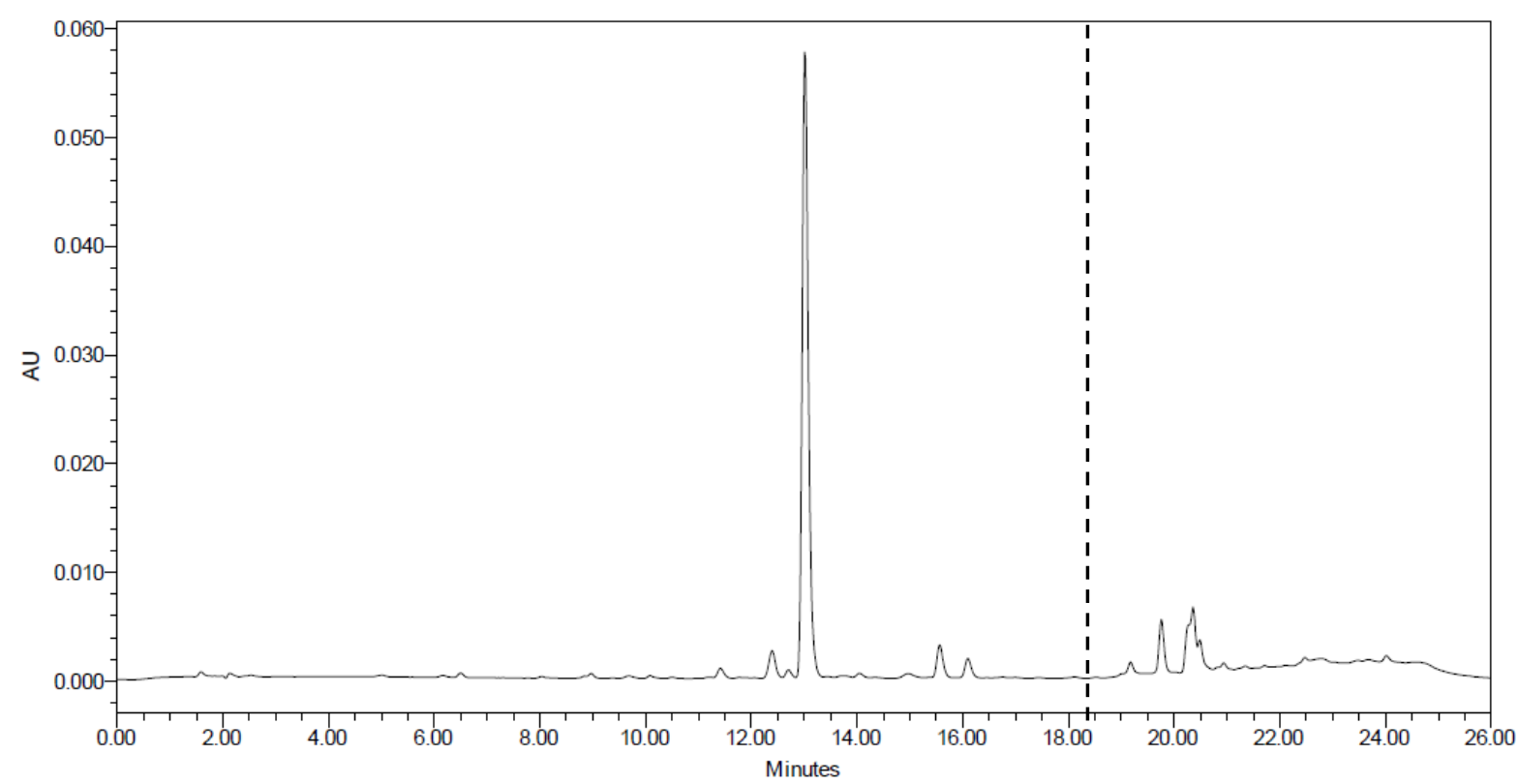

Figure S29. RP-HPLC Chromatogram of the crude obtained after coupling with preactivation of 5 min.

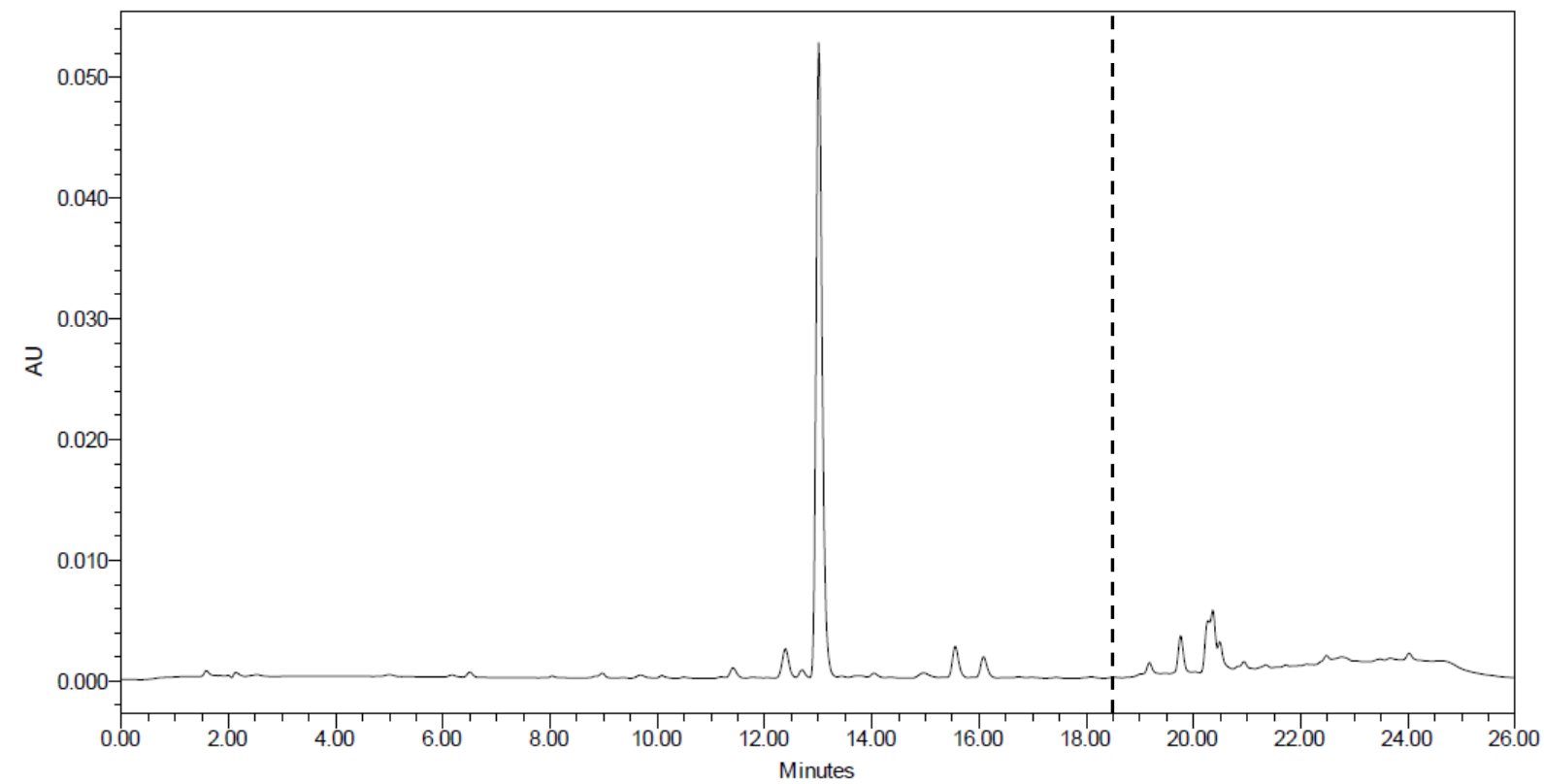

Figure S30. RP-HPLC Chromatogram of the crude obtained after coupling with preactivation of $10 \mathrm{~min}$. 


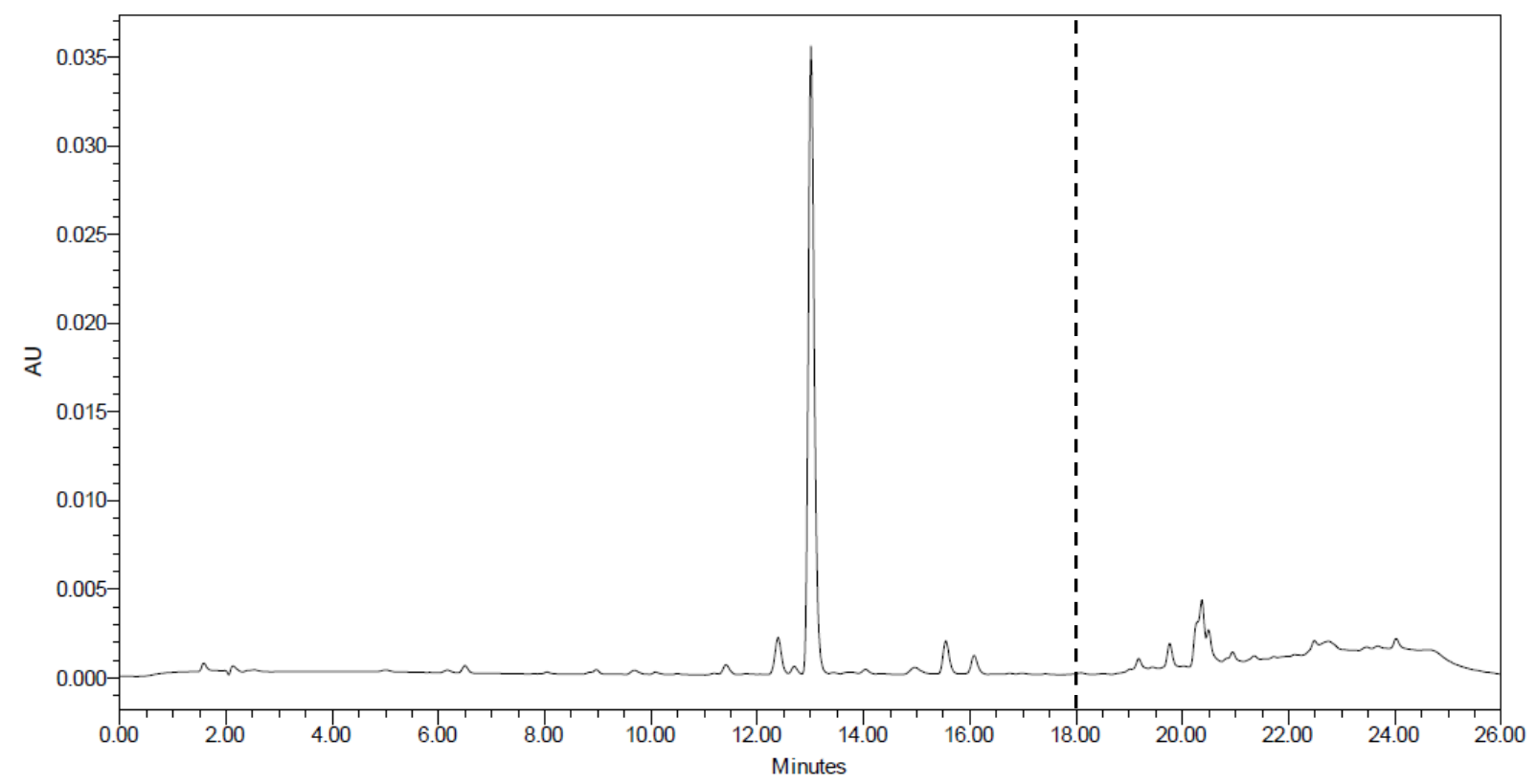

Figure S31. RP-HPLC Chromatogram of the crude obtained after coupling with preactivation of 20 min.

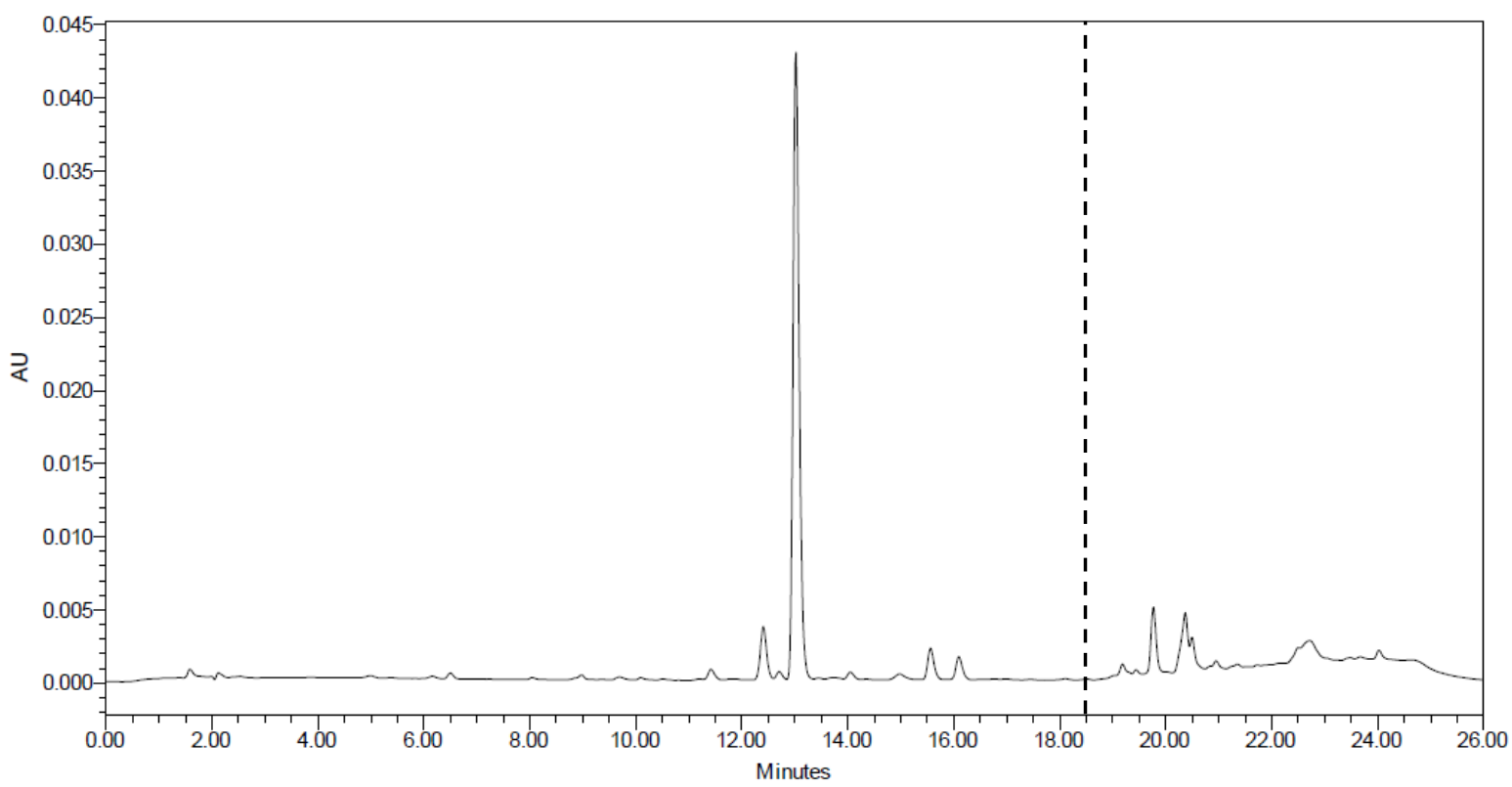

Figure S32. RP-HPLC Chromatogram of the crude obtained after coupling with preactivation of 60 min. 
3.11 HPLC Results for the effect of duration during preactivation with COMU as coupling agent

Peak at 12.5 minutes corresponds to peptide $\mathbf{1}$ and peak at 13 minutes corresponds to the product 2. All chromatograms were recorded for crude samples after cleavage without further purification. Peaks after 18 minutes (designated by the vertical dashed line) are related to residual protecting group moieties and to the HPLC gradient program, which corresponds to washing region and do not contain the peptides that are relevant for this study.

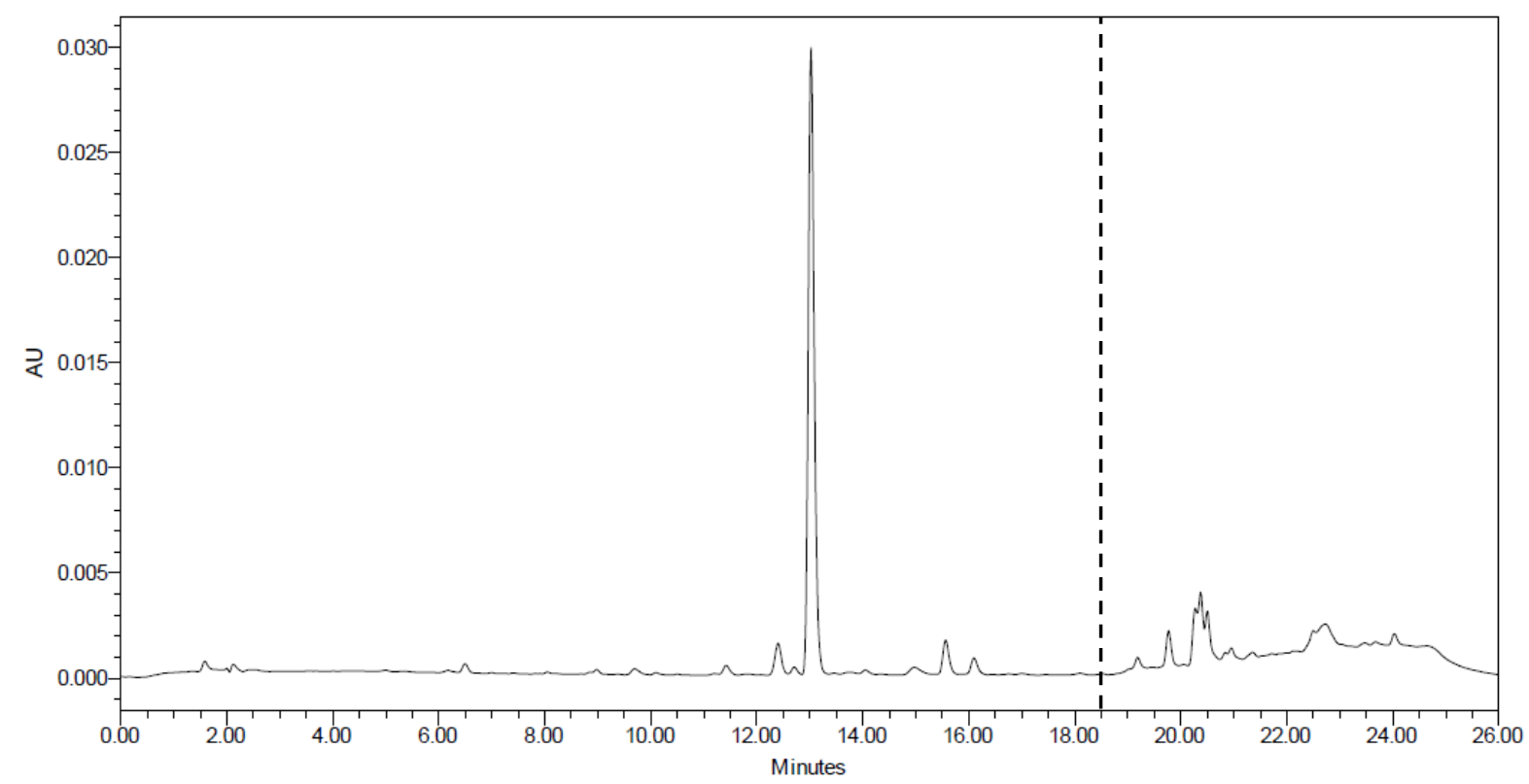

Figure S33. RP-HPLC Chromatogram of crude obtained after coupling with preactivation of $1 \mathrm{~min}$. 


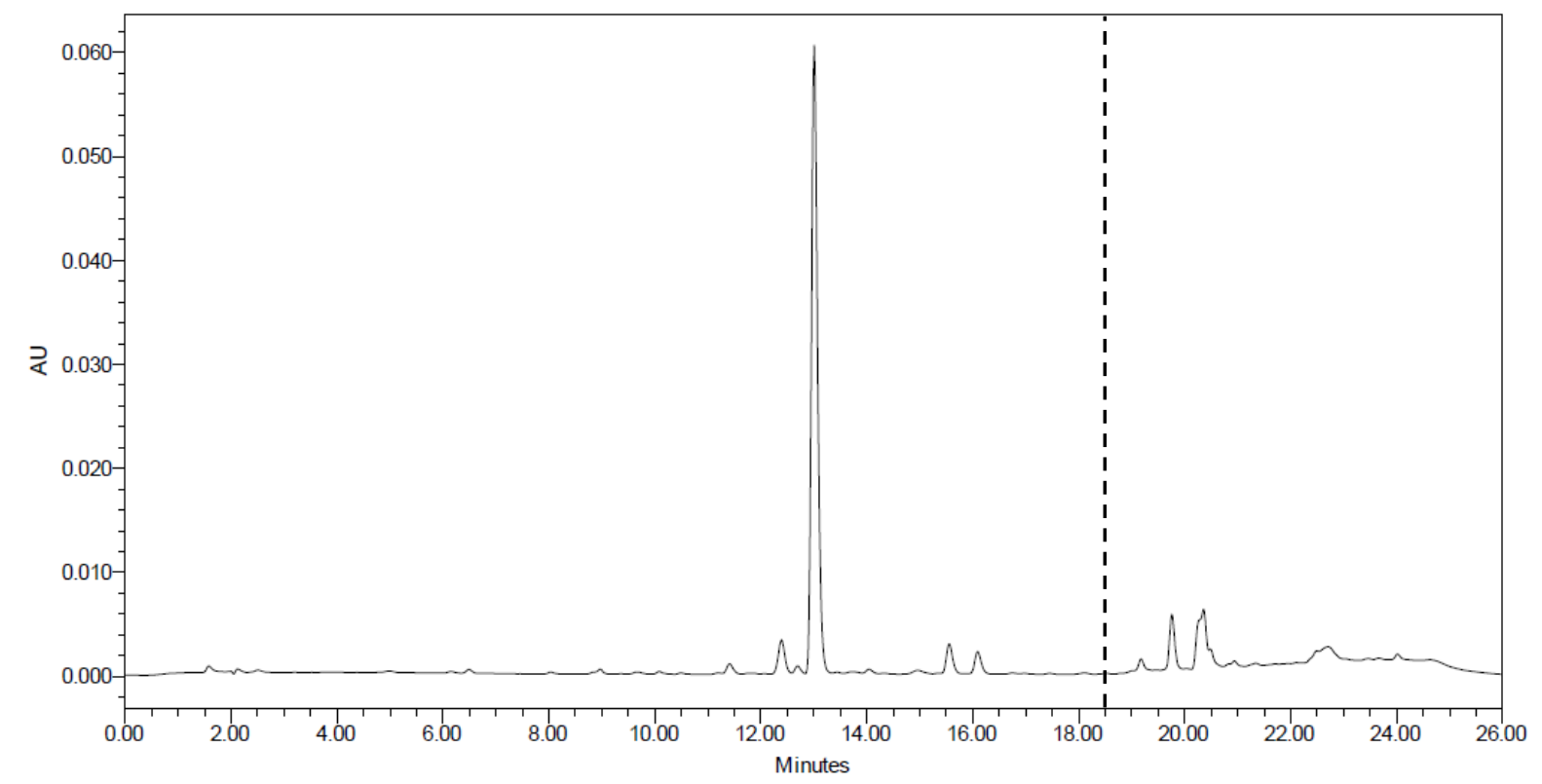

Figure S34. RP-HPLC Chromatogram of the crude obtained after coupling with preactivation of 5 min.

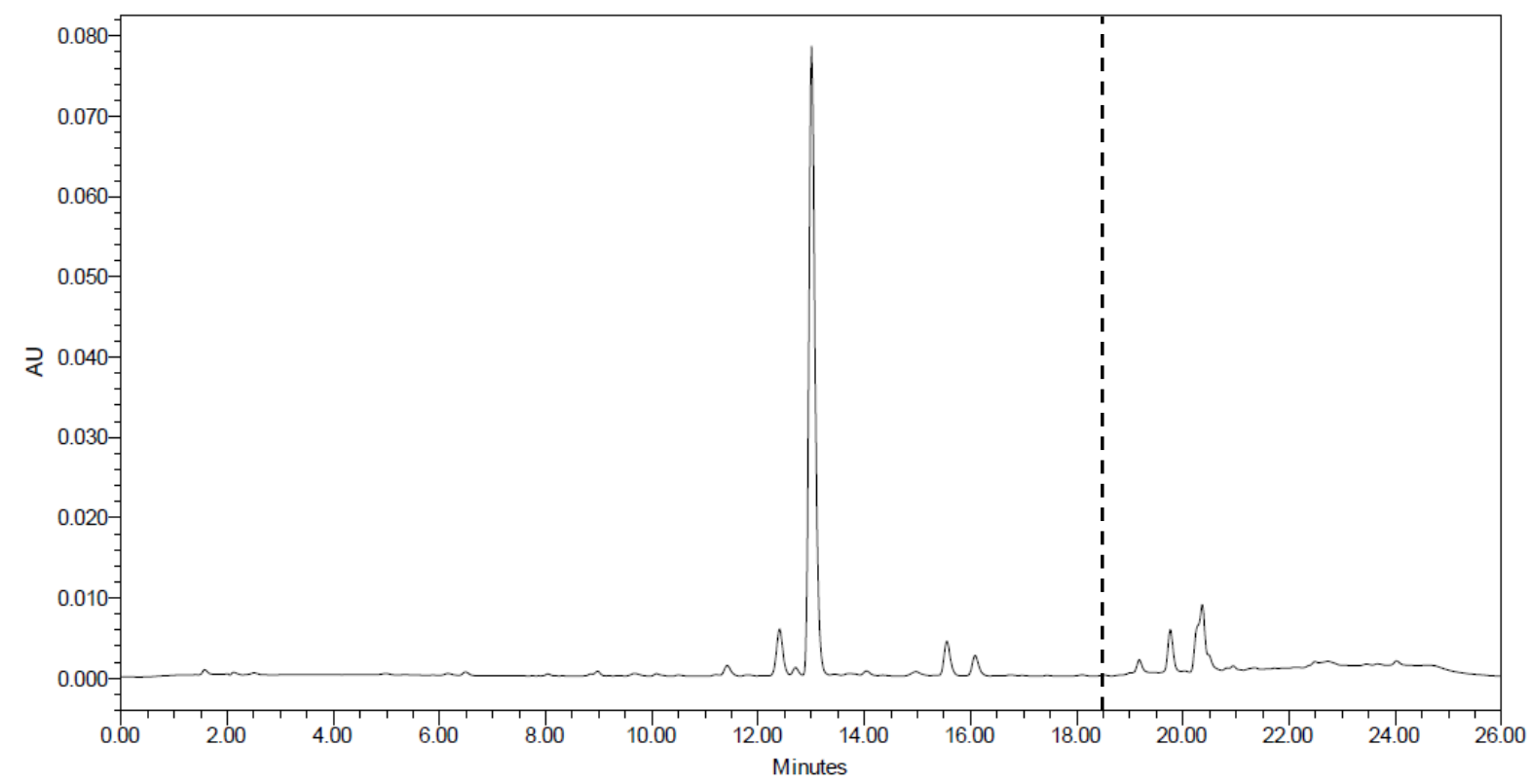

Figure S35. RP-HPLC Chromatogram of the crude obtained after coupling with preactivation of 10 min. 


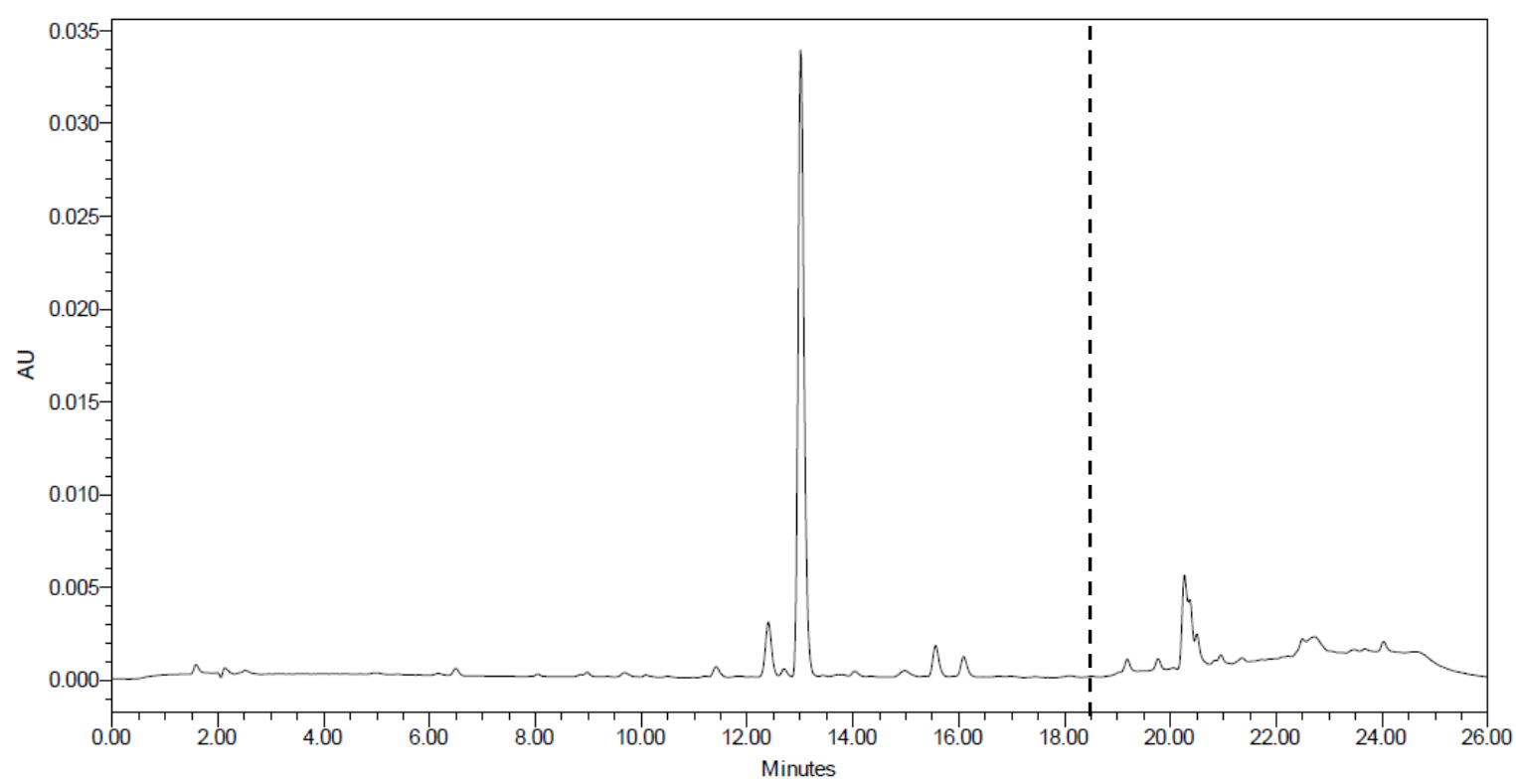

Figure S36. RP-HPLC Chromatogram of crude obtained after coupling with preactivation of 20 min.

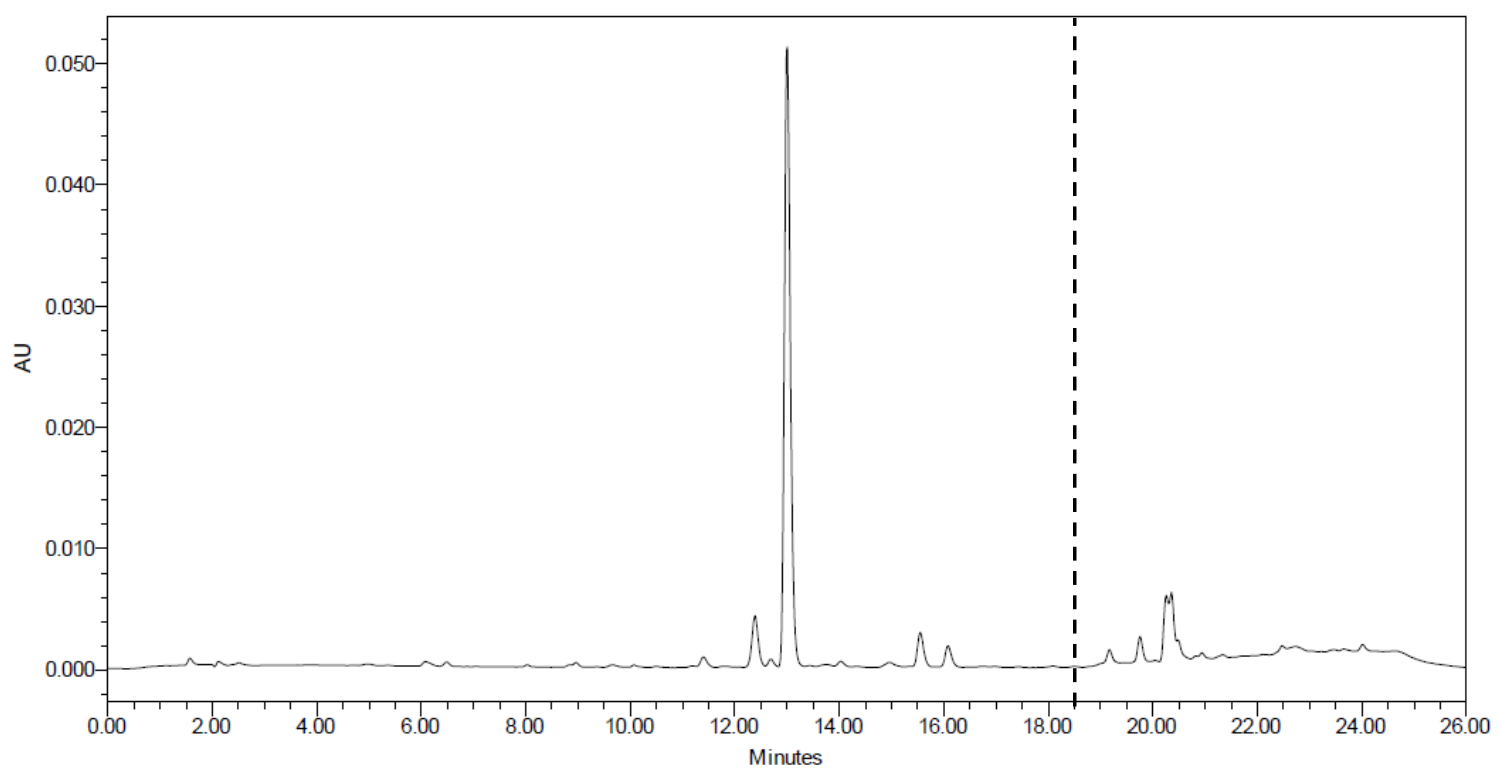

Figure S37. RP-HPLC Chromatogram of crude obtained after coupling with preactivation of 40 min. 


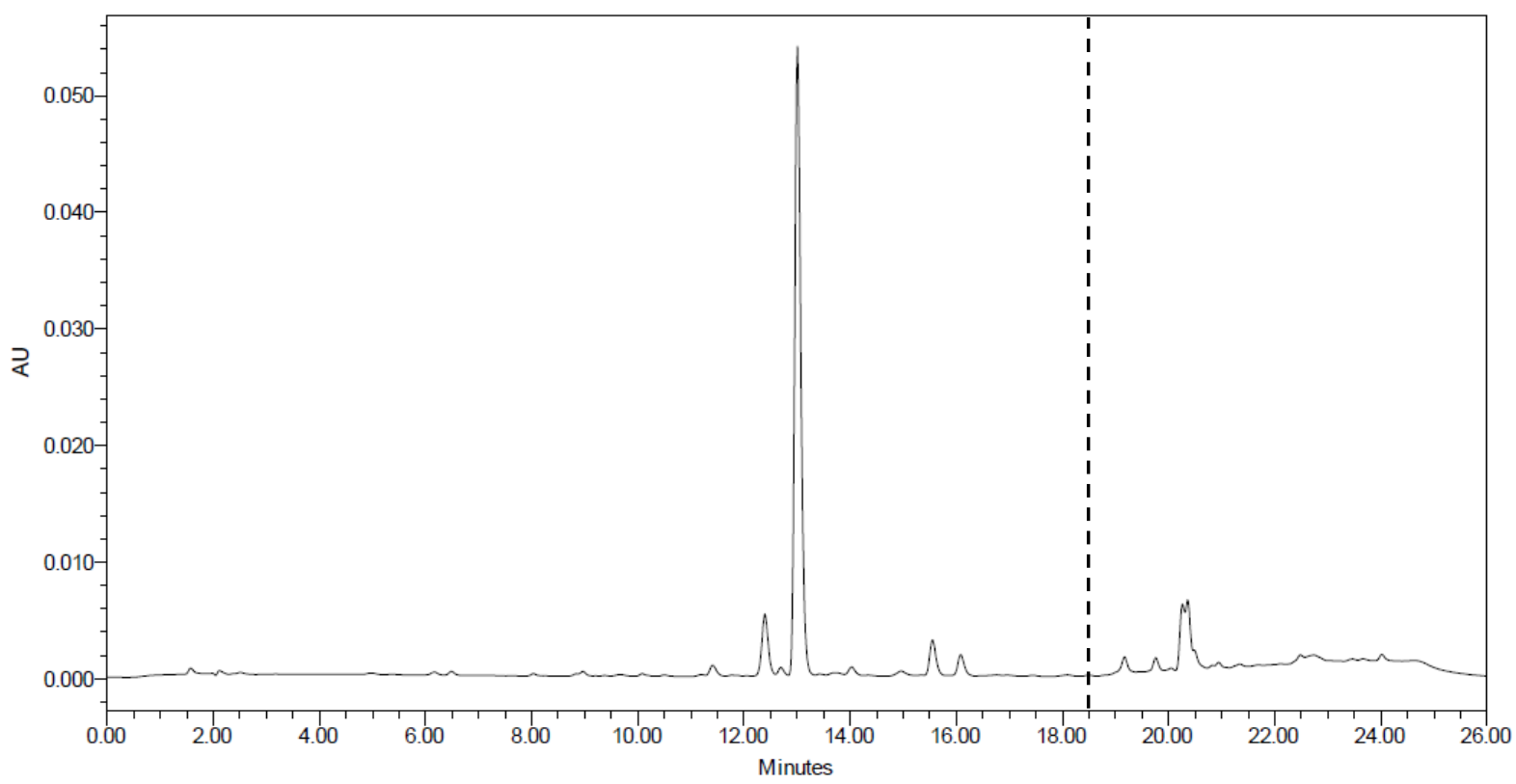

Figure S38. RP-HPLC Chromatogram of the crude obtained after coupling with preactivation of $60 \mathrm{~min}$. 


\subsection{HPLC Results of the synthesis of SOM}

The peak at 10 minutes corresponds to the desired product SOM. The peaks at 8 minutes and at 14.5 minutes correspond to the deletion sequences des-Trp-SOM and des-Lys-SOM respectively. Peaks after 18 minutes (designated by the vertical dashed line) are related to residual protecting group moieties and to the HPLC gradient program, which corresponds to washing region and do not contain the peptides that are relevant for this study.

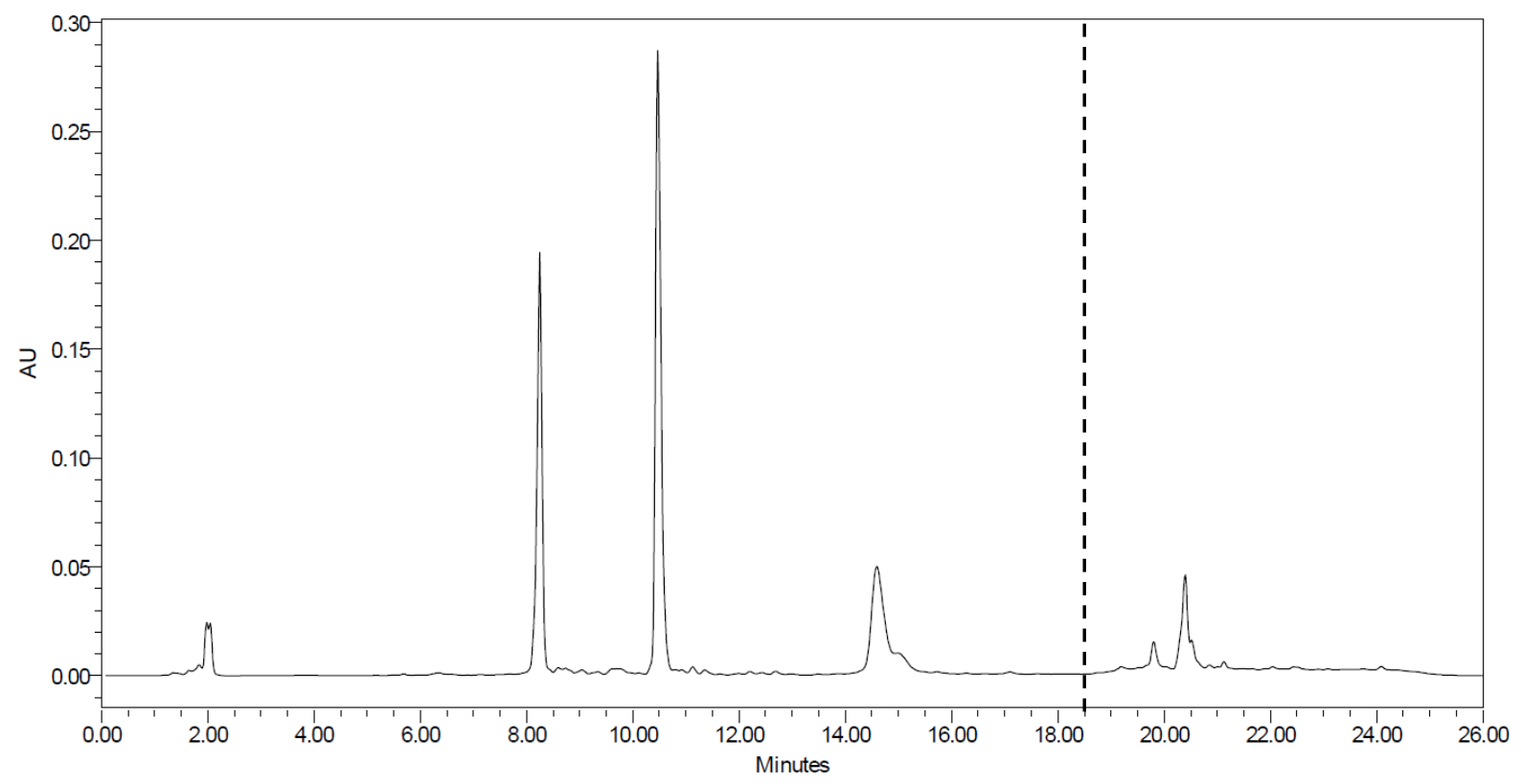

Figure S39. RP-HPLC Chromatogram of the crude obtained after SOM 1 synthesis applying 1.5 MER in a shaker. 


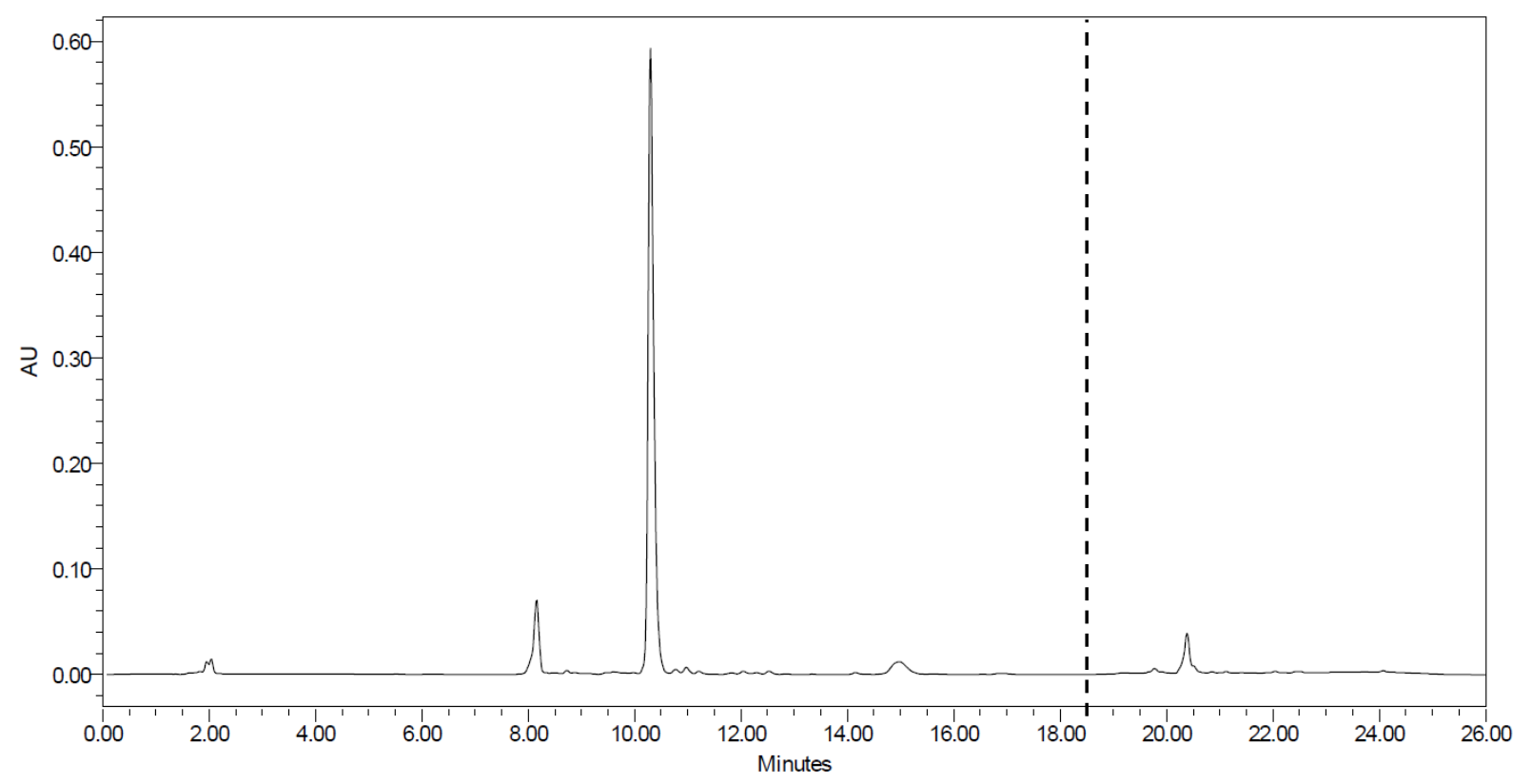

Figure S40. RP-HPLC Chromatogram of the crude obtained after SOM 2 synthesis applying 3 MER in a shaker.

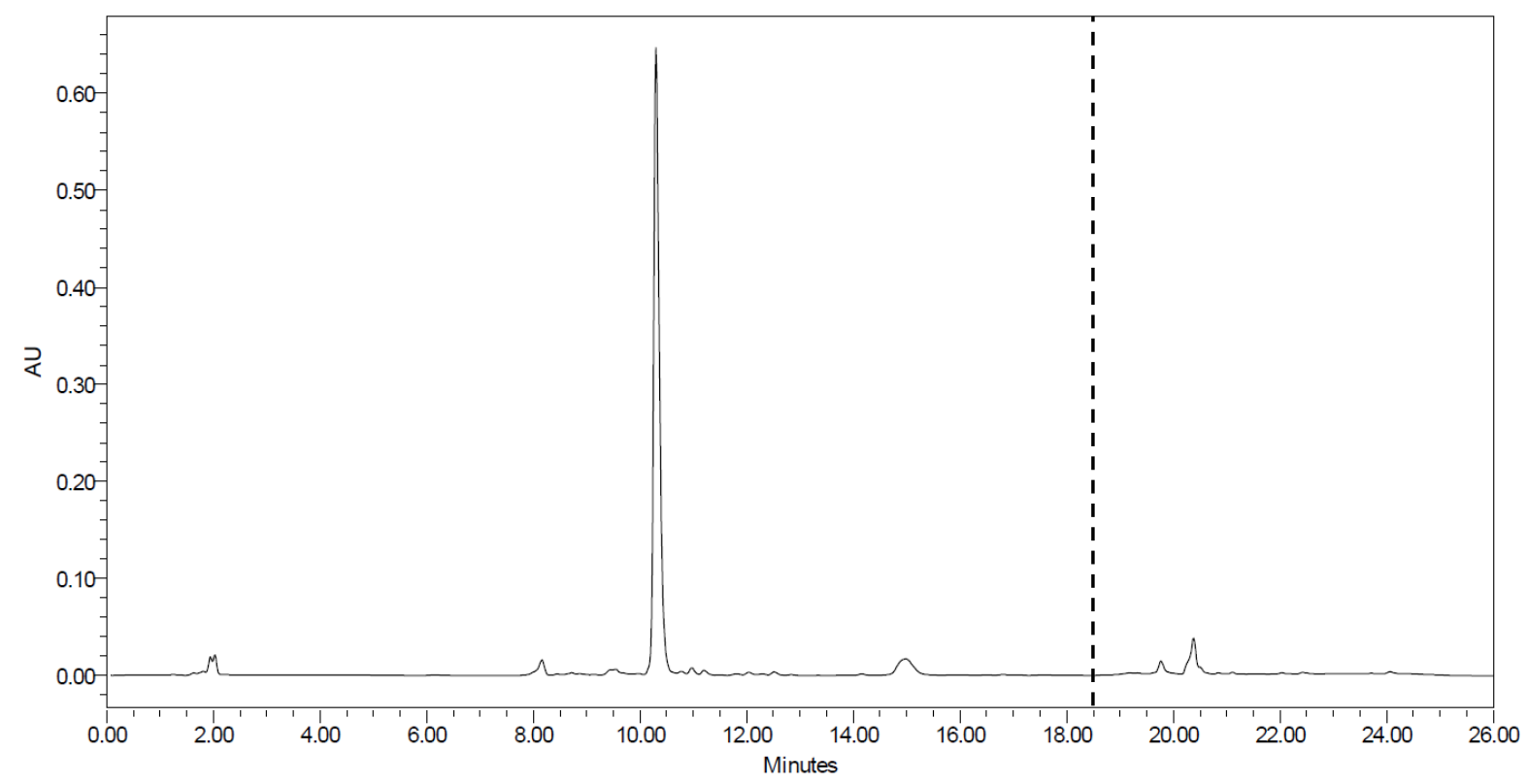

Figure S41. RP-HPLC Chromatogram of the crude obtained after SOM 3 synthesis applying 1.5 MER in HSS. 


\subsection{HRMS of SOM}

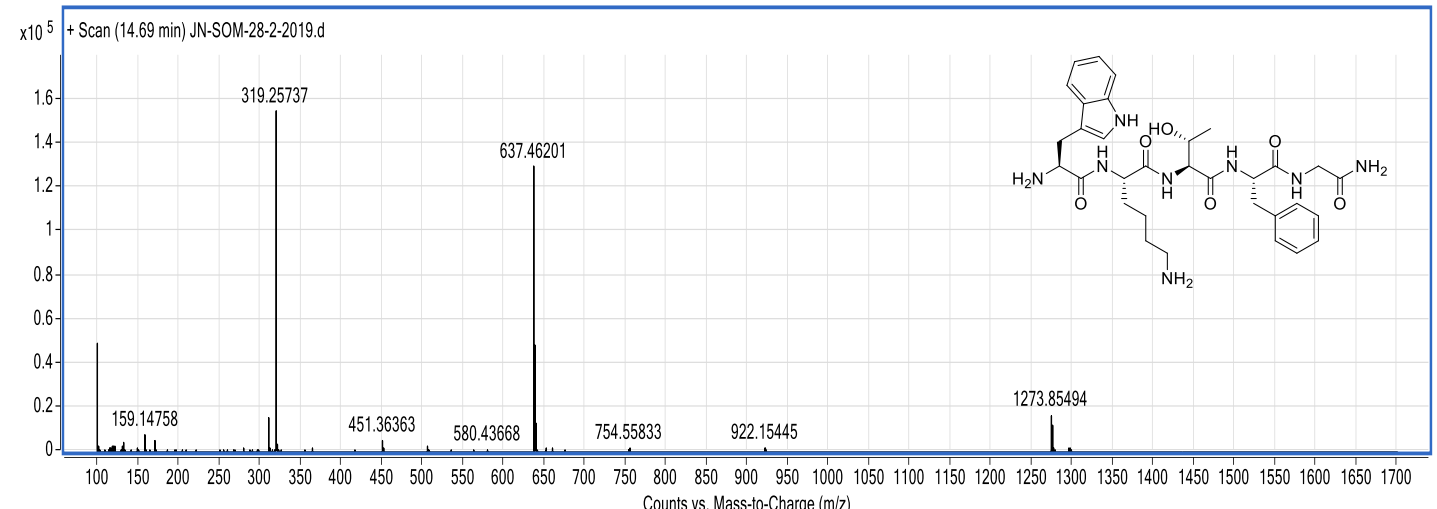

Figure S42. HRMS of the peak at 8 minutes (Figure S39) with observed $\mathrm{m} / \mathrm{z}$ of $637.46,319.25$ and 1273.85 corresponding to calculated des-Trp-SOM m/z $+\mathrm{H}^{+}, 0.5 \mathrm{~m} / \mathrm{z}+\mathrm{H}^{2+}$, and $2 \mathrm{~m} / \mathrm{z}+\mathrm{H}^{+}$respectively

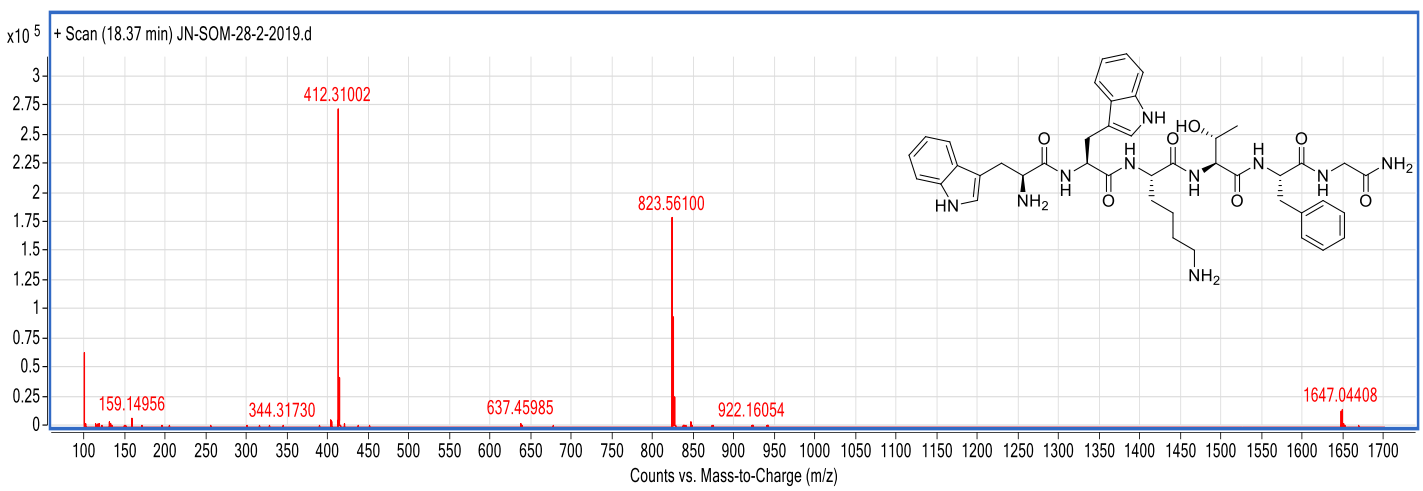

Figure S43. HRMS of the peak at 10.5 minutes (Figure S39) with observed $\mathrm{m} / \mathrm{z}$ of $823.56,412.31$, 1647.04 corresponding to calculated $\mathbf{S O M ~ m} / \mathrm{z}+\mathrm{H}^{+}, 0.5 \mathrm{~m} / \mathrm{z}+\mathrm{H}^{2+}$, and $2 \mathrm{~m} / \mathrm{z}+\mathrm{H}^{+}$respectively.

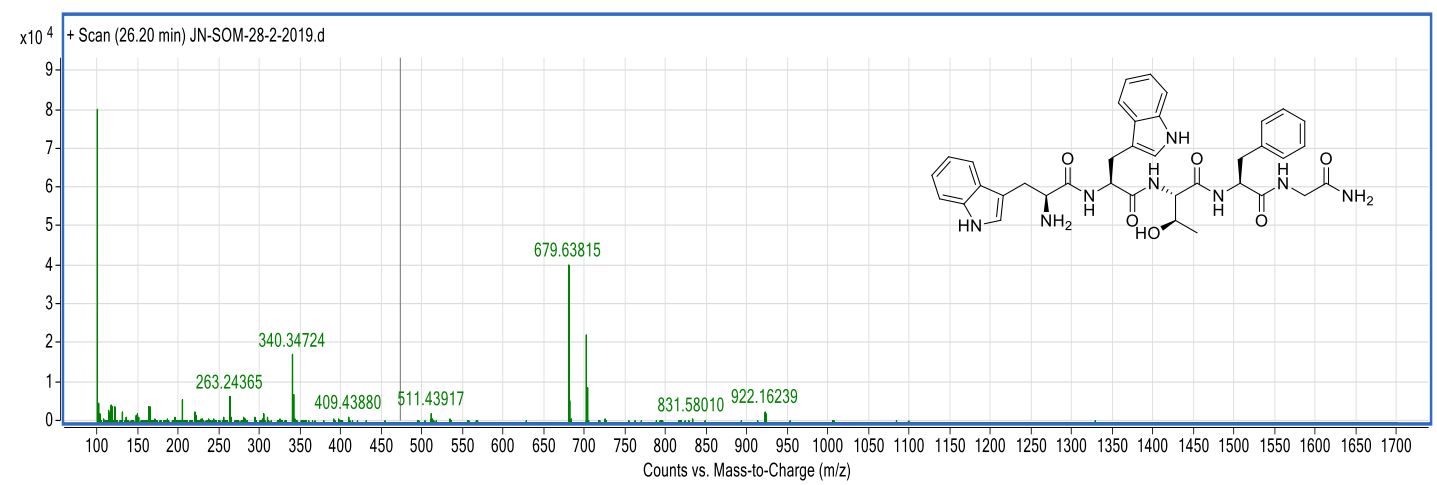

Figure S44. HRMS of the peak at 14.5 minutes (Figure S39) with observed $\mathrm{m} / \mathrm{z}$ of 679.64 corresponding to calculated des-Lys-SOM $\mathrm{m} / \mathrm{z}-\mathrm{NH}_{3}+\mathrm{H}^{+}$. 


\subsection{Microscope images of beads after applying HSS}

All the microscope pictures were taken with the same zoom of X 500. The average diameter of the resin is approximately $100 \mu \mathrm{m}$. The scale bar is the same for all images.

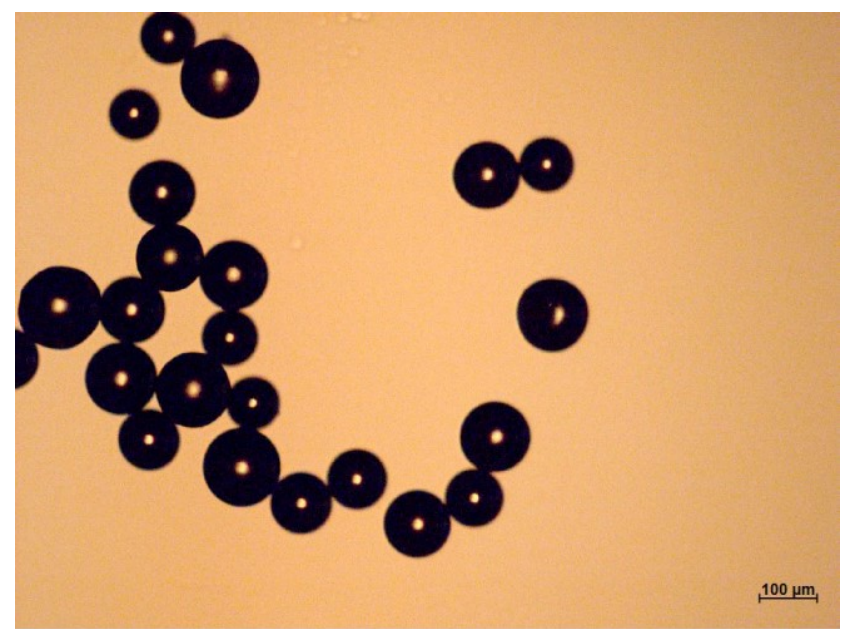

Figure S45. Image of the polystyrene beads presenting the integrity and shape of the beads, taken after the coupling of $\mathbf{4 a}$ to peptide $\mathbf{1}$ to produce peptide $\mathbf{4}$ by stirring via HSS at $900 \mathrm{rpm}$.

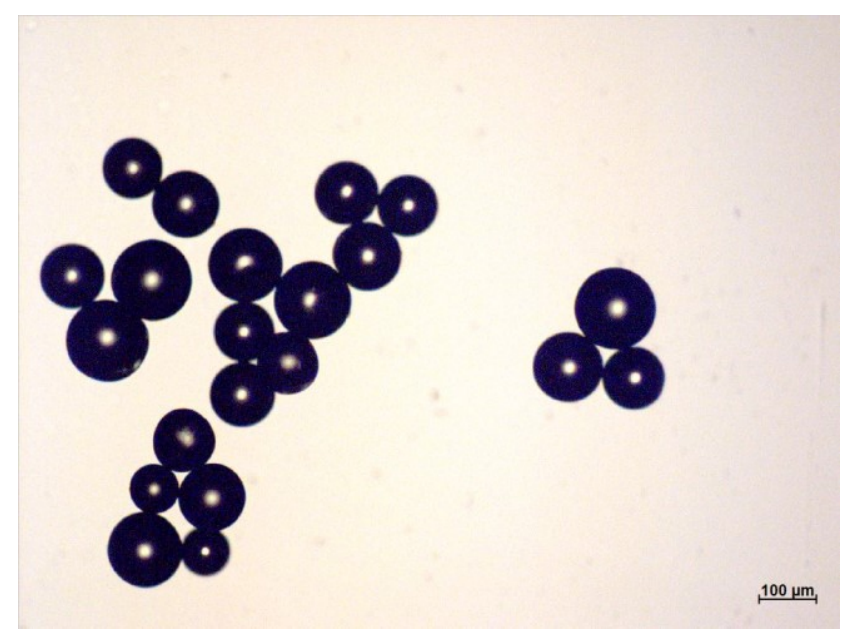

Figure S46. Image of the polystyrene beads presenting the integrity and shape of the beads, taken after the coupling of $\mathbf{4 a}$ to peptide $\mathbf{1}$ to produce peptide $\mathbf{4}$ by stirring via HSS at $700 \mathrm{rpm}$ for $2 \mathrm{~h}$. 


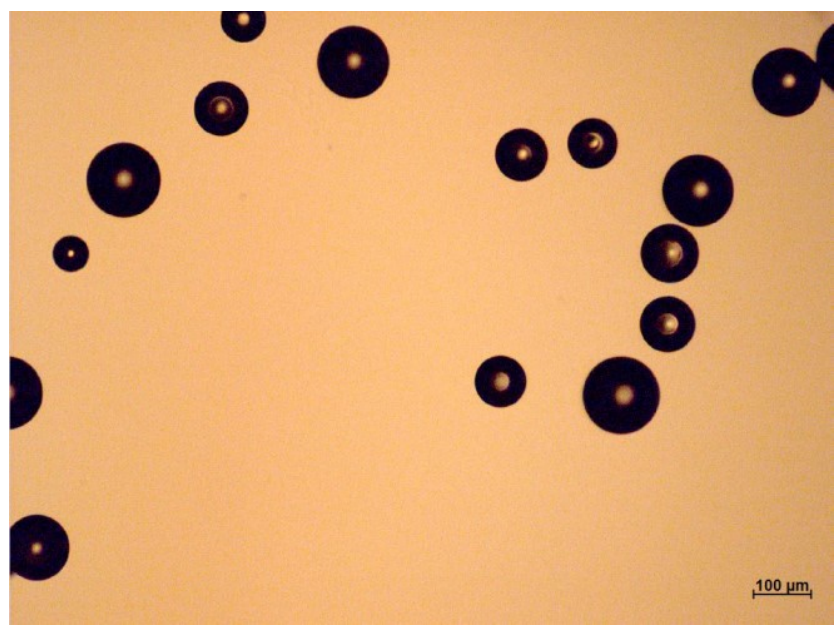

Figure S47. Image of the polystyrene beads presenting the integrity and shape of the beads, taken after the SOM 3 synthesis stirring via HSS at $700 \mathrm{rpm}$.

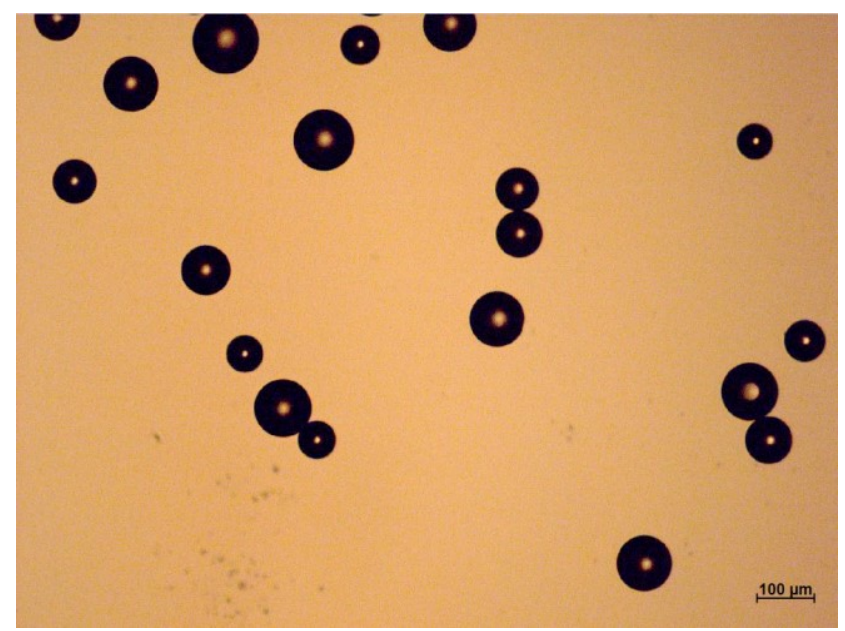

Figure S48. Image of the dry polystyrene beads that was taken before swelling. 
3.15 HPLC analysis of Mixture of 4 (D-His), 4 (L-His) and 1

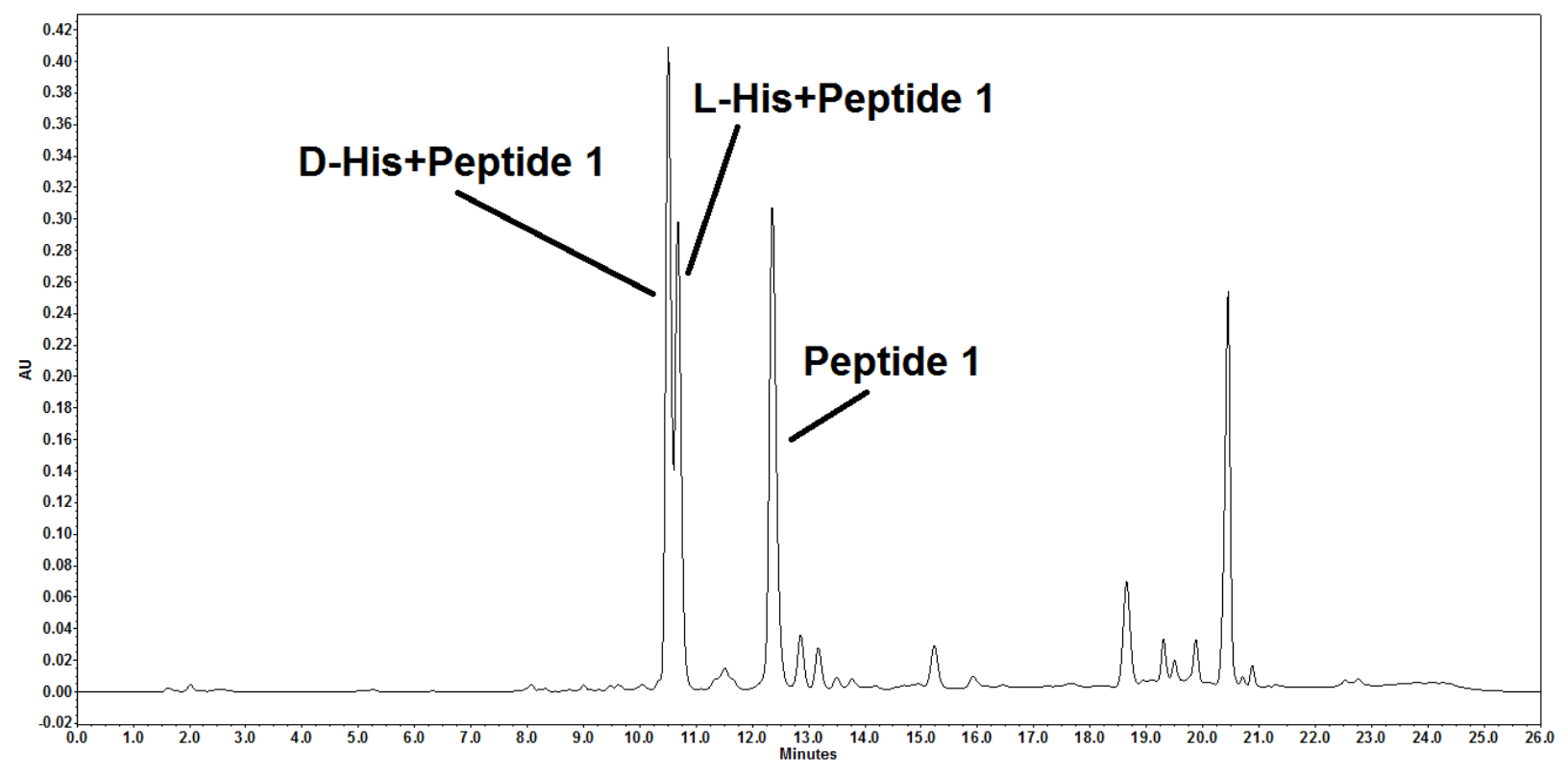

Figure S49. RP-HPLC Chromatogram of the crude Mixture of independently synthesized peptides that are: i) peptide 1 ii) peptide 1 after coupling with L-His iii) peptide 1 after coupling with D-His. 